\title{
Cultural Assimilation: Learning and Sorting
}

\author{
STEIN MONTEIRO* ${ }^{\dagger}$ \\ Ryerson University
}

\begin{abstract}
Immigration from poorer source countries is larger than from richer countries, so that poor country immigrants have greater exposure to co-ethnics, leading to fewer incentives to learn the local culture and assimilate. In this paper, the exposure channel through which source country richness affects assimilating immigration is modelled through neighbourhood location choices and incentives to learn the local culture in the host country. Two equilibrium outcomes are identified, in which, there is either only assimilating immigration in at least one neighbourhood of the host country (sorting equilibrium) when immigration is from a rich source country, or there is some non-assimilating immigration in all neighbourhoods (mixed equilibrium) when immigration is from a poor source country. The presence of this exposure channel is tested using data from the Longitudinal Survey of Immigrants in Canada: waves 1-3. Learning, rather than sorting into co-ethnic communities, is the main factor operating in the exposure channel between source country richness and assimilating immigration.
\end{abstract}

Keywords: Cultural Assimilation; Language Proficiency; Pre-immigration Experience; Ethnic Enclaves; Sorting; Exposure.

JEL Classifications: J15, Z13

\section{Introduction}

Immigrants exhibit strong location preferences in the host country. New immigrants tend to locate close to existing migrant networks and where there are existing concentrations of immigrant groups, called ethnic enclaves. The presence of migrant networks explains to a large degree the location choices of new immigrants in the host country (Nowotny \& Pennerstorfer, 2019; Pedersen, Pytlikova \& Smith, 2008). In turn the location of immigrants determines their patterns of cultural and economic assimilation (Massey, 1985). New immigrants living among co-ethnics have lower levels of English language skill acquisition (Allen \& Turner, 1992; Beckhusen, Florax, Graaff, Poot \& Waldorf, 2012; Danzer \& Yaman, 2016; Danzer, Feuerbaum,

*I would like to acknowledge the following people for their comments and suggestions that contributed to improvements in this study: Sam Bucovetsky, Uros Petronijevic, Benjamin Sand, Tasso Adamopolous, Nippe Lagerlöf, Ida Ferrara, Akram Panahi Dar-gahloo, Andrew Hencic, Andrew Dickens, Chiara Marazzi, Imran Aziz, Michelle Tong, Rachel Harris, Sara Tumpane, Shafkat Ali, Vajiheh Mahdiyan Amirabadi, and an anonymous reviewer.

Contact: steim@ryerson.ca

(C) 2021 Stein Monteiro. Licenced under the Creative Commons Attribution-Noncommercial 4.0 Licence (http://creativecommons.org/licenses/by-nc/4.0/). Available at http://rofea.org. 
Piopiunik \& Woessmann, 2018) and earnings (Allen \& Turner, 1992; Warman, 2006; Xie \& Gough, 2011; Damm, 2009). But enclaves also serve as a means to minimize costs in communication with co-ethnics, and the cost of transportation from the consumption of ethnic goods (Chiswick \& Miller, 2005). Living in an ethnic enclave is essential for new immigrants beginning the integration process into host country culture. Enclaves provide benefits to new immigrants through the social networks embedded within the enclave. In addition, low skilled immigrants living in an enclave earn about $13 \%$ more than comparable immigrants outside the enclave (Edin, Fredriksson \& Aslund, 2003). This is indicative that new immigrants have higher returns to foreign experience and education inside the enclave (Xie \& Gough, 2011; although this effect varies across immigrant groups). Finally, enclaves are places of entrepreneurialism where individuals start ethnic businesses (Portes \& Shafer, 2006). Clearly, there is mixed evidence to support the view that the enclave can be a useful resource to new immigrants through its strong network links, but may also encumber new immigrants in their learning of the local culture. However, it remains that a language barrier continues to exist for new immigrants and the enclave is a means to alleviate some of the communication frictions that exist between new immigrants and native-born.

New immigrants face cultural barriers that make communication with native-born difficult. Given that communication with others is an integral part of a productive society, authorities in major immigrant receiving country are increasingly interested in issues surrounding immigrant assimilation because faster assimilation and/or close integration with native-born are assumed to be beneficial for all. Alberto, Johann \& Rapoport (2013) show increasing birthplace diversity among all persons living in major immigrant receiving countries such as USA, UK, Austria, Norway, Germany, Belgium and Canada between 1990 and 2000 (see table 1). On the other hand, the birthplace among migrants of these countries did not become much more diverse in the ten years $1990-2000^{1}$. In fact, the USA grew in total immigration and became less diverse. This indicates that immigrants are a growing proportion of the population but are being selected from fewer source countries. In 2011, Canada had a foreign-born population of $6,775,800$ people ( $20.6 \%$ of the total population). The highest proportion among the G8 countries. Asia (including the Middle East) was Canada's largest source of immigrants over the five years 2006-2011. Although the share of immigration from Africa, Caribbean, Central and South America increased slightly. Canada appears to be becoming more diverse in its source country selection (Statistics Canada, 2013). Ultimately, governments are not interested in removing all immigration; some immigration is worthwhile and preferred. In the sphere of immigration policy design, the type of immigration is of greater concern.

\footnotetext{
${ }^{1}$ Except for New Zealand. New Zealand took in a lot of new immigrants and from a variety of source countries.
} 


\section{MONTEIRO Cultural Assimilation: Learning and Sorting}

Table 1: Ethnic, linguistic and changes in birthplace diversity indices across major immigrant receiving countries

\begin{tabular}{lcccc}
\hline \hline & & & \multicolumn{2}{c}{ \% change in birthplace diversity 1990-2000 } \\
\cline { 3 - 5 } Country & Ethnic & Linguistic & All & Migrants \\
\hline Australia & 0.093 & 0.335 & $-4 \%$ & $3 \%$ \\
Austria & 0.107 & 0.152 & $119 \%$ & $3 \%$ \\
Belgium & 0.555 & 0.541 & $15 \%$ & $2 \%$ \\
Canada & 0.712 & 0.577 & $8 \%$ & $1 \%$ \\
France & 0.103 & 0.122 & $-1 \%$ & $1 \%$ \\
Germany & 0.168 & 0.164 & $33 \%$ & $1 \%$ \\
Netherlands & 0.514 & 0.335 & $2 \%$ & $2 \%$ \\
Norway & 0.059 & 0.067 & $46 \%$ & $1 \%$ \\
New Zealand & 0.397 & 0.166 & $8 \%$ & $3 \%$ \\
Switzerland & 0.531 & 0.544 & $0 \%$ & $-3 \%$ \\
USA & 0.490 & 0.251 & $40 \%$ & $2 \%$ \\
UK & 0.121 & 0.053 & $21 \%$ & $17 \%$ \\
\hline \hline
\end{tabular}

Source: Alesina, Alberto; Harnoss, Johann \& Rapoport, Hillel (2015). Birthplace Diversity and Economic Prosperity. Journal of Economic Growth, Vol. 21, issue 2 (June 2016), pp. 101-138.

Increasing diversity will invariably be accompanied by increasing communication frictions between new immigrants and native-born. In this paper, I model how sorting and/or learning among new immigrants alleviates those frictions. The size of the co-ethnic group combined with clustering into co-ethnic communities determine the level of exposure to co-ethnics that new immigrants will experience in the host country. Choosing to live in a neighbourhood that is predominately co-ethnic reduces communication costs for the new immigrant, but compromises integration into the host country culture (i.e. the assimilating decision). Overtime, ethnic enclaves may be viewed as a "training system" for new immigrants before entering the mainstream economy. The training system is a composite of labour market information, recruitment practices and skill accumulation. The enclave is an institution of formal and informal traditions that are different from the mainstream sectors of the economy. New immigrants will choose to live in enclaves as a means to minimize communication costs while learning the local culture (Bailey \& Waldinger, 1991; and Waldinger, 1993). Eventually immigrants learn the local language. Preliminary evidence suggests that exposure to co-ethnics is associated with lower assimilation rates in the short-run. Using the 2001 Canadian Census of Population and focusing on the sub-sample of immigrants with no English speaking background, that is, immigrants from non-English speaking households whose mother tongue is non-English, 18-64 years old, and do not reside in Quebec. New immigrants that have lived in Canada for under a year, some are living within enclaves consisting of predominately co-ethnics. The preliminary evidence suggests that $81.68 \%$ of those living in an ethnic enclave ${ }^{2}$ spoke English and $83.37 \%$ of those living outside the enclave did speak English. A difference of $-1.69 \% * *$ (0.0064). Among im-

${ }^{2}$ Construction of the ethnic enclave variable is discussed further in the empirical section. 
migrants that have lived in Canada for 2 years the difference increases to $-2.71 \% * * *(0.0031)$. Among immigrants that have lived in Canada for 5 years the difference decreases drastically to $-0.84 \% * *(0.0029)$, then $-0.50 \% *(0.0028)$ after living in Canada for 10 years. In the short-run, there are growing differences in the composition of immigration inside ethnic enclaves compared to outside. Eventually, these differences disappear as immigrants learn the local culture through formal or informal means.

The model developed in this paper extends the work of Lazear (1999) and Konya (2007) by including a spatial dimension to account for the differences in communication frictions across neighbourhoods. In the model, potential migrants from the sending country are randomly matched with other immigrants and native-born in the host country. Due to productivity differences between the source and host country, immigration from poorer countries is expected to be larger than from richer ones. Figure 1 supports this claim, depicting a negative relationship between source country RGDP per capita (i.e. a proxy for richness of the source country) in 2000 and the total number of new immigrants in Canada from the 2001 Canadian Census of Population. Since the share of immigrants will be larger from a poorer country, these migrants will be exposed to co-ethnics more often. In turn, the higher exposure to co-ethnics will decrease assimilation rates for immigrants from the poorer country. Given that the only source of friction in this model is finding a partner to communicate with and all surplus created is through communicating effectively, the distribution of immigrants across neighbourhoods matter, as are the subsequent incentives from learning the local culture within each neighbourhood. Since location choices are non-random, this would imply multiple equilibrium outcomes with varying levels of clustering, exposure to co-ethnics and assimilation rates across neighbourhoods.

The two channels of sorting and learning are interacting. Related empirical research to support the findings of this paper is the evidence on substitutability between sorting into co-ethnic communities and learning the local culture. Bauer, Epstein \& Gang (2005) provide evidence from the US that Mexican migrants with improved English language proficiency choose smaller networks (i.e. they rely less on the ethnic enclave). Similarly, a more recent study by Bredtmann, Nowotny Otten (2020) using within-country regional data from the European Union shows that the ability to communicate in the host country language (measured by linguistic distance) and network size are substitutes, such that linguistic distance and size of the linguistic network jointly determine location choices in the host country. The substitution between sorting into ethnic enclaves and learning the local culture explains the finding in the empirical section of the paper.

The empirical section of this paper uses data from the Longitudinal Survey of Immigrants in Canada: waves 1-3 where cultural assimilation is measured by proficiency in English, investment into native-born culture is measured by formal/informal learning of English, and location in an ethnic enclave is determined jointly by use of an unofficial language at work and clustering of co-ethnics in the CMA/CA of arrival. I provide estimates of source country 


\section{MONTEIRO Cultural Assimilation: Learning and Sorting}

richness on cultural assimilation through the exposure channel to test the implications of the theoretical model. I find that sorting and/or learning is an important component of the channel through which source country richness affects assimilation rates. However, controlling for formal/informal learning, source country richness does not have any significant effect on assimilating immigration. This would indicate that sorting, independent from learning, is not a major component of the relationship between source country richness and assimilating immigration. I test whether sorting is the only thing being estimated by over-adjusting for the implied sorting effect. By over-adjusting I intentionally include a proxy for co-ethnic sorting (a measure for living/working in an ethnic enclave) into the regression model. Source country richness now has a significant positive effect on assimilating immigration. This says that there is an alternate channel unaccounted for by the current model through which source country richness affects assimilation rates. I show that this alternate channel functions prior to sorting and is probably related to quality of the immigrant group rather than size of immigration.

In the next section I formulate a simplified model that incorporates location choice among neighbourhoods in the host country and show that multiple equilibria are possible. In section 3 of the paper I empirically test these findings.

\section{Model}

The mechanism functioning to transmit personal and contextual characteristics into language proficiency are generalized as exposure, incentives and efficiency (van Tubergen \& Kalmijn, 2009). Exposure and incentives function interactively to determine the level of cultural integration. However, exposure is determined by location preferences of new immigrants upon arrival. Selection into a neighbourhood must be introduced into the model to better understand the relationship between exposure and language accumulation. Exposure is typically measured by the size of the immigrant group. An increase in the size of the immigrant group decreases incentives to learn the local culture. These exposure effects are stronger within ethnic enclaves leading to shifts in the composition of immigration within the enclave and outside of it. I will present a theoretical model accounting for these features and derive the equilibria that arise from this environment.

Communication frictions between native-born and immigrants are modelled into the host country environment through a random matching framework. I assume there are only two countries in the world: North and South, where the South is less developed relative to the North. Thus migration flows from South to North. The North is further segmented into neighbourhoods, but for simplicity I assume that there are only two neighbourhoods. There are two sets of agents: native-born and immigrants. The native-born agents are located in the North and potential migrants are located in the South. The potential migrants in the South make a decision about whether to stay in the South or migrate to the North. Additionally, potential migrants must also decide whether to assimilate or not, and simultaneously choose which neighbourhood 

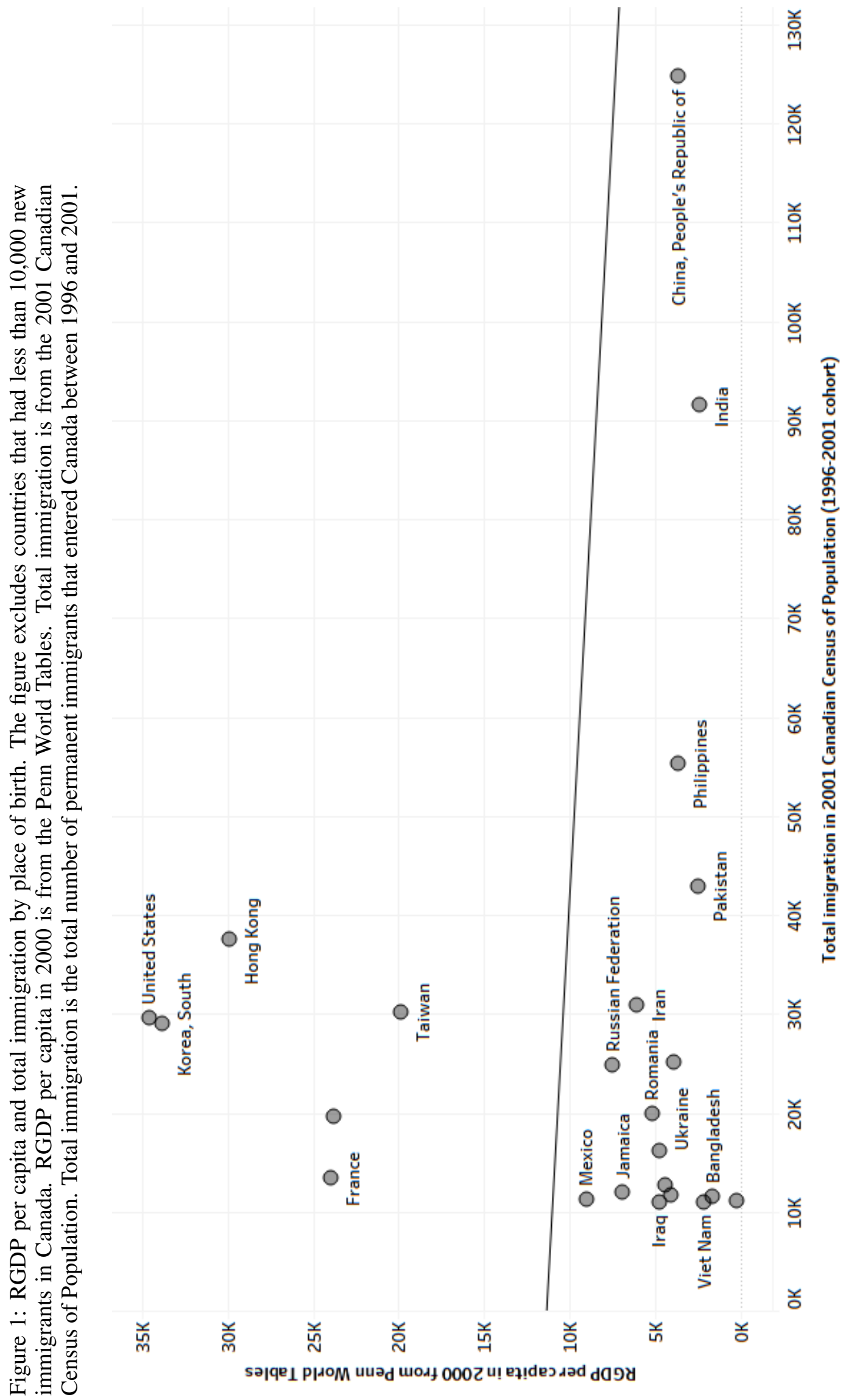


\section{MONTEIRO Cultural Assimilation: Learning and Sorting}

in the North to live and work. The location choice depends on the relative size of their networks across the neighbourhoods of the North. Potential migrants choose a strategy that gives the best outcome based on a set of individual-specific characteristics.

Individuals in the North are drawn together randomly so that matches are created. Efficient matching is the primary mode of production. The model introduces communication frictions that inhibit ease of production by randomly matching agents. A match between persons of similar culture are able to generate a surplus but matches between persons of different culture create no surplus. Matches between a non-assimilating and a native-born create no surplus while matches between a non-assimilating and an assimilating immigrant, or an assimilating immigrant and a native-born, do generate a surplus. The randomness in meeting people of common cultures are effectively reduced in the presence of location choice. Potential migrants that fail to assimilate choose less communication frictions by living in neighbourhoods with a large number of immigrants. As new immigrants enter the host country their location decisions are determined by the immigrant distribution and in turn influence their learning decision. In this model the location decision of other new immigrants is influenced by the location and learning decisions of other immigrant cohorts simultaneously. Equilibrium is achieved when no individual has an incentive to relocate from their current neighbourhood and their learning decisions are optimized.

\subsection{Thresholds}

In this section I will discuss further the potential migrant's decision problem, how heterogeneity is introduced into the model, derive the equilibrium thresholds for migrating and assimilating, and discuss the equilibria that come out of the random matching immigration problem. The migration cost is

$$
\text { Migration cost }=\mu c
$$

where $\mathrm{c}$ is an individual-specific migration cost that is distributed over all potential migrants in the South, $c \sim F(c)$ and $c \in[0,1]$. And $\mu<1$ is an index of physical distance between the North and the South. Migration costs do not differ across neighbourhoods in the North. Potential migrants may face assimilation costs of

$$
\text { Assimilation cost }=\theta v \iota
$$

where $\theta<1$ is an index of cultural differences between the North and the South and $\iota$ is investment into cultural assimilation by unit cost of investing $v$. Assimilation costs are determined by the level of investment that is undertaken. Investment is a part of the process of cultural capital accumulation that is either amassed over time in a passive learning process where immigrants learn about the host country's culture through time spent in the host country. Or, formal 
and informal investments may be made towards learning the culture as a decisive step towards integration.

Learning is introduced through the capital accumulation process. Learning is distinguished at their pre- and post-immigration levels where pre-immigration experience with the local culture is an individual-specific distribution in the source country, while post-immigration learning comes as a form of investment into learning the local culture after the migration decision has been made. Further learning in the host country are individual-specific decisions that are a function of the investment returns and costs.

Cultural capital has two components, $x=\phi_{1} \iota+\phi_{2} \omega$ where $x \in[0,1]$ is the total number of native-born that the migrant can communicate with given the level of investment $\iota$ and preimmigration experience $\omega$. The parameters $\phi_{1}, \phi_{2} \geq 0$ are constant conversion factors of preand post-immigration experience into the units of $x$. Alternatively, $\phi_{1}$ and $\phi_{2}$ may be interpreted as the returns to further learning and pre-immigration experience. For simplicity, I suppose that $\phi_{1}=\phi_{2}=1$. Cultural capital is dichotomous; immigrants can either talk to all native-born $(x=1)$ or some $(x=\omega)$. Pre-immigration experience $\omega$ is accumulated capital or endowment. Accumulated capital $\omega$ is distributed $W(\omega), \omega \in[0,1]$. It represents accumulated learning that includes the potential migrant's experience with host country culture prior to migrating. Investment is a dichotomous variable representing further learning, $\iota \in\{0,1\}$. New immigrants with large pre-immigration experience ( $\omega$ is large) face the lowest cost of investment $(\iota=1-\omega)$.

Assimilating immigrants can generate surplus with other immigrants or native-born. Nonassimilating immigrants can generate surplus with other immigrants but the chance of meeting another immigrant depends on the neighbourhood they will choose to live in and the size of their network. Suppose there are two neighbourhoods $i=A, B$ such that the potential migrant's neighbourhood choice is given by a general preference parameter, $\beta \sim B(\beta)$ and $\beta \in[0,1]$, which is an individual-specific variable measuring the size of the immigrant's network in neighbourhood $i$ relative to neighbourhood $j .^{3}$ New immigrants with a large network in $i=A, B$ prefer this neighbourhood over any other $j \neq i$.

The assimilating immigrant can trade with anyone within their own neighbourhood and earn a surplus of one, but must incur the cost of migration and assimilation. New immigrants with the largest $\omega$ have the lowest assimilating cost and the largest incentive to learn the local culture. Assimilating immigrants are not limited to trading with other immigrants, they can also trade with native-born. However, assimilating immigrants are limited to trading within the neighbourhood. The non-assimilating immigrant only trades with other immigrants within the neighbourhood and face no assimilation cost. All meetings with Southerners will produce a surplus of $h$ with no migration and assimilation costs incurred. Those that stay in the South will generate a surplus of $h<1$ (this condition ensures that migration only moves from South to North; that is, matches are more efficient in the North than in the South). The potential

\footnotetext{
${ }^{3}$ This formulation is similar to cultural segregation in a variant of the model presented in Bonn (2012a).
} 
migrant's decisions are summarized by the following set of value functions

$$
\begin{aligned}
V_{a}^{i} & =\beta(1-\theta v(1-\omega)-\mu c) \\
V_{a}^{j} & =(1-\beta)(1-\theta v(1-\omega)-\mu c) \\
V_{n}^{i} & =\beta\left(\frac{m^{i}}{1+m^{i}}-\mu c\right) \\
V_{n}^{j} & =(1-\beta)\left(\frac{m^{j}}{1+m^{j}}-\mu c\right) \\
V_{s} & =h
\end{aligned}
$$

where $i, j=A, B$ and $j \neq i$, the total number of native-born in neighbourhood $i$ is normalized to one, and $m^{i} \in[0, \infty)$ is the total number of immigrants in neighbourhood $i=A, B$. Equations (1) and (2) are the utility from assimilating immigration within neighbourhood $i, j=A, B$ and $j \neq i$. Equations (3) and (4) are the utility from non-assimilating immigration within neighbourhood $i, j=A, B$ and $j \neq i$. Equation (5) is the utility from not migrating.

Equating (1) and (3), and solving for $\omega$ derives the threshold level for assimilating in neighbourhood $i=A, B$, such that $\omega \geq \omega_{a}$ there is assimilating immigration, and non-assimilating immigration otherwise. There are two seperate threshold conditions for each $i=A, B$.

$$
\begin{aligned}
& \omega_{a}^{i}=1-\frac{1}{\theta v\left(1+m^{i}\right)}, \\
& \omega_{a}^{j}=1-\frac{1}{\theta v\left(1+m^{j}\right)},
\end{aligned}
$$

where $i, j=A, B$ and $j \neq i$. Among those in the assimilating group, the neighbourhood choice is given by the threshold $\beta_{a}$. The location choice for the assimilating group is derived by equating (1) and (2), and solving for $\beta$, as such

$$
\beta_{a}=1 / 2
$$

Those assimilating immigrants with $\beta \geq \beta_{a}$ there is immigration to neighbourhood $i$, otherwise neighbourhood $j$. Similarly among non-assimilating immigrants, the neighbourhood choice is derived by equating (3) and (4), and solving for $\beta$ (or for $c$ ). This is the threshold $\beta_{n}(c)$ (or $c\left(\beta_{n}\right)$ )

$$
\begin{aligned}
\beta_{n}(c) & =\frac{m^{j} /\left(1+m^{j}\right)-2 \mu c}{m^{i} /\left(1+m^{i}\right)+m^{j} /\left(1+m^{j}\right)-2 \mu c}, \\
\text { or } c\left(\beta_{n}\right) & =\frac{m^{j} /\left(1+m^{j}\right)-\left(m^{i} /\left(1+m^{i}\right)\right)\left(\beta_{n} /\left(1-\beta_{n}\right)\right)}{2 \mu}
\end{aligned}
$$

Non-assimilating immigrants with $\beta>\beta_{n}(c)$ will choose to live in neighbourhood $i$, others will choose neighbourhood $j$. In addition to threshold conditions (6) - (9) which describe the individual's assimilation and location incentives, the following four conditions determine the 
individual's migration choices which must also be satisfied. Equating (5) to either (1) or (2) provides the threshold for assimilating immigration to neighbourhood $i$ or $j$, respectively. Similarly, equating (5) to either (3) or (4) is the threshold for non-assimilating immigration to neighbourhood $i$ or $j$, respectively. The set of immigration thresholds are

$$
\begin{aligned}
c_{a}^{i}(\omega, \beta) & =\frac{1-\theta v(1-\omega)-h / \beta}{\mu} \\
c_{a}^{j}(\omega, \beta) & =\frac{1-\theta v(1-\omega)-h /(1-\beta)}{\mu} \\
c_{n}^{i}(\beta) & =\frac{m^{i} /\left(1+m^{i}\right)-h / \beta}{\mu} \\
c_{n}^{j}(\beta) & =\frac{m^{j} /\left(1+m^{j}\right)-h /(1-\beta)}{\mu}
\end{aligned}
$$

Only those Southerners with small migration costs will migrate. Non-assimilating immigration to neighbourhood $i=A, B$ must satisfy $c \leq c_{n}^{i}(\beta), \omega<\omega_{a}^{i}$ and $\beta>\beta_{n}(c)$. Non-assimilating immigration to neighbourhood $j$ must satisfy $c \leq c_{n}^{j}(\beta), \omega<\omega_{a}^{j}$ and $\beta<\beta_{n}(c)$. These conditions for the non-assimilating group may be plotted in $(c, \beta)$ space (see figure 2). Similarly, assimilating immigration to neighbourhood $i=A, B$ must satisfy $c \leq c_{a}^{i}(\omega, \beta), \omega>\omega_{a}^{i}$, and $\beta>\beta_{a}=1 / 2$. Assimilating immigration to neighbourhood $j$ must satisfy $c \leq c_{a}^{j}(\omega, \beta), \omega>\omega_{a}^{j}$, and $\beta<\beta_{a}=1 / 2$. Figure 3 shows these conditions for the assimilating group in $(c, \beta)$ space assuming $\omega>\omega_{n}^{i}$.

It is more useful to view the decision space for both groups in a single diagram. However, this requires one of the two variables, $\omega$ or $\beta$, to be held constant while the other is graphed as a function of $c$. I have chosen to hold $\beta$ constant and depict the various regions in $(c, \omega)$ space. Note that the assimilating immigration thresholds $c_{a}^{i}(\omega, \beta)$ and $c_{a}^{j}(\omega, \beta)$ are a function of $\beta$ and $\omega$. For given values of $\beta$, the two functions are related as follows

$$
c_{a}^{i}(\omega, \beta) \begin{cases}>c_{a}^{j}(\omega, \beta) & \text { if } \beta>1 / 2 \\ =c_{a}^{j}(\omega, \beta) & \text { if } \beta=1 / 2 \\ <c_{a}^{j}(\omega, \beta) & \text { if } \beta<1 / 2\end{cases}
$$

Figure 4 depicts the non-assimilating and assimilating immigration groups for different levels of $\omega$ and $c$ in the case when $\beta>1 / 2$. A similar graph exists for $\beta<1 / 2$ which would depict assimilating immigration to neighbourhood $j$. Non-assimilating immigration to neighbourhood $j$ must satisfy the condition that $c \leq c_{n}^{j}(\beta), \omega<\omega_{a}^{j}$ and $\beta<\beta_{n}(c)$. The first two conditions are identifiable in figure 4, but the last condition is not a function of $\omega$. To be able to plot this condition in $(c, \omega)$ space insert $\beta_{n}(c)$ into $c_{a}^{j}(\omega, \beta)$ and solve for $c$. The result is

$$
c_{a}^{j}\left(\omega, \beta_{n}(c)\right) \equiv c_{n}^{j}(\omega)=\frac{m^{i} /\left(1+m^{i}\right)(1-\theta v(1-\omega))-h\left(m^{i} /\left(1+m^{i}\right)+m^{j} /\left(1+m^{j}\right)\right)}{\mu\left(m^{i} /\left(1+m^{i}\right)-2 h\right)}
$$


MONTEIRO Cultural Assimilation: Learning and Sorting

Figure 2: Non-assimilating immigration; $m^{j} \geq m^{i}$

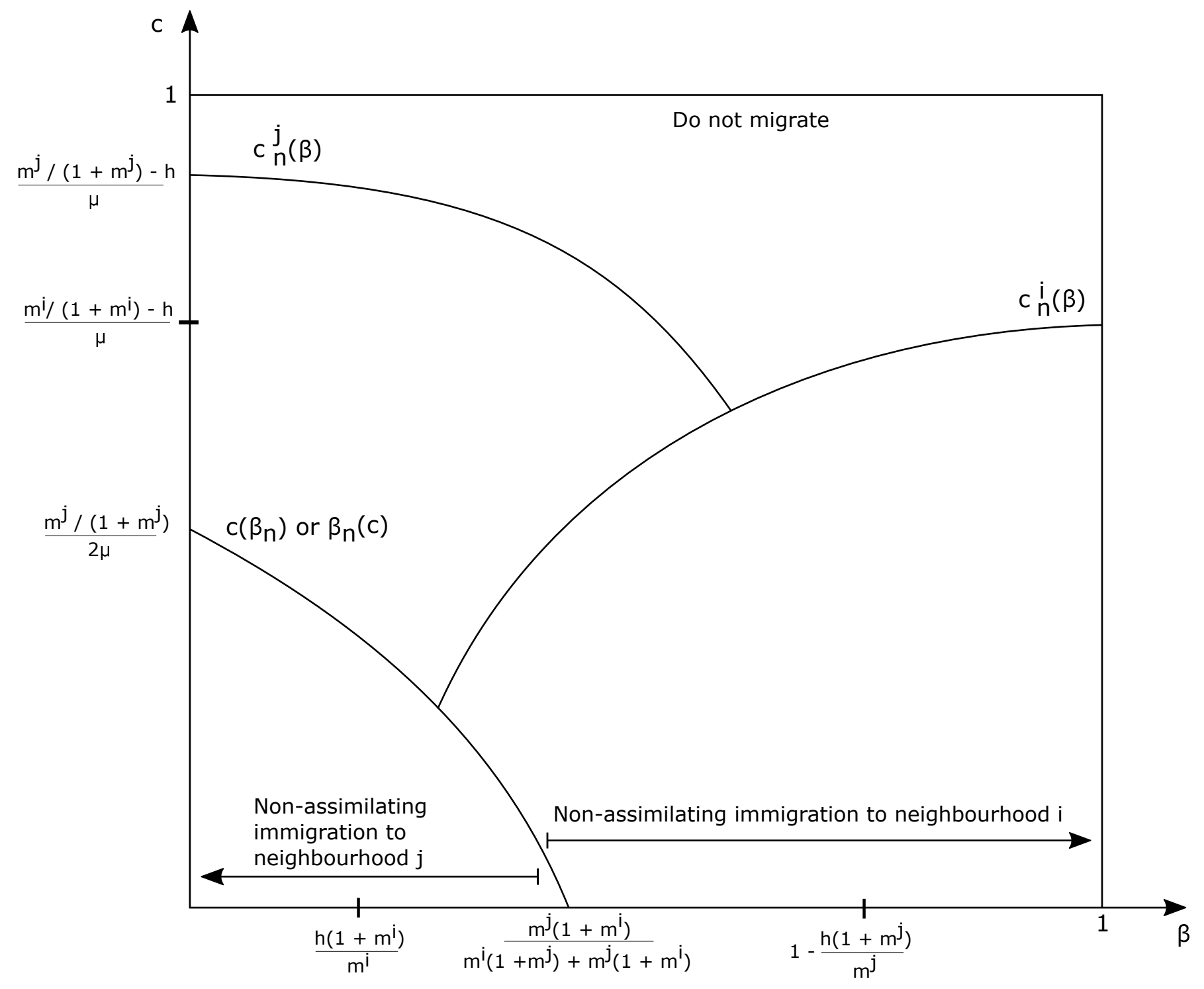


Review of Economic Analysis 13 (2021) 115-156

Figure 3: Assimilating immigration; $m^{j} \geq m^{i}$ and $\omega>\omega_{n}^{i}$

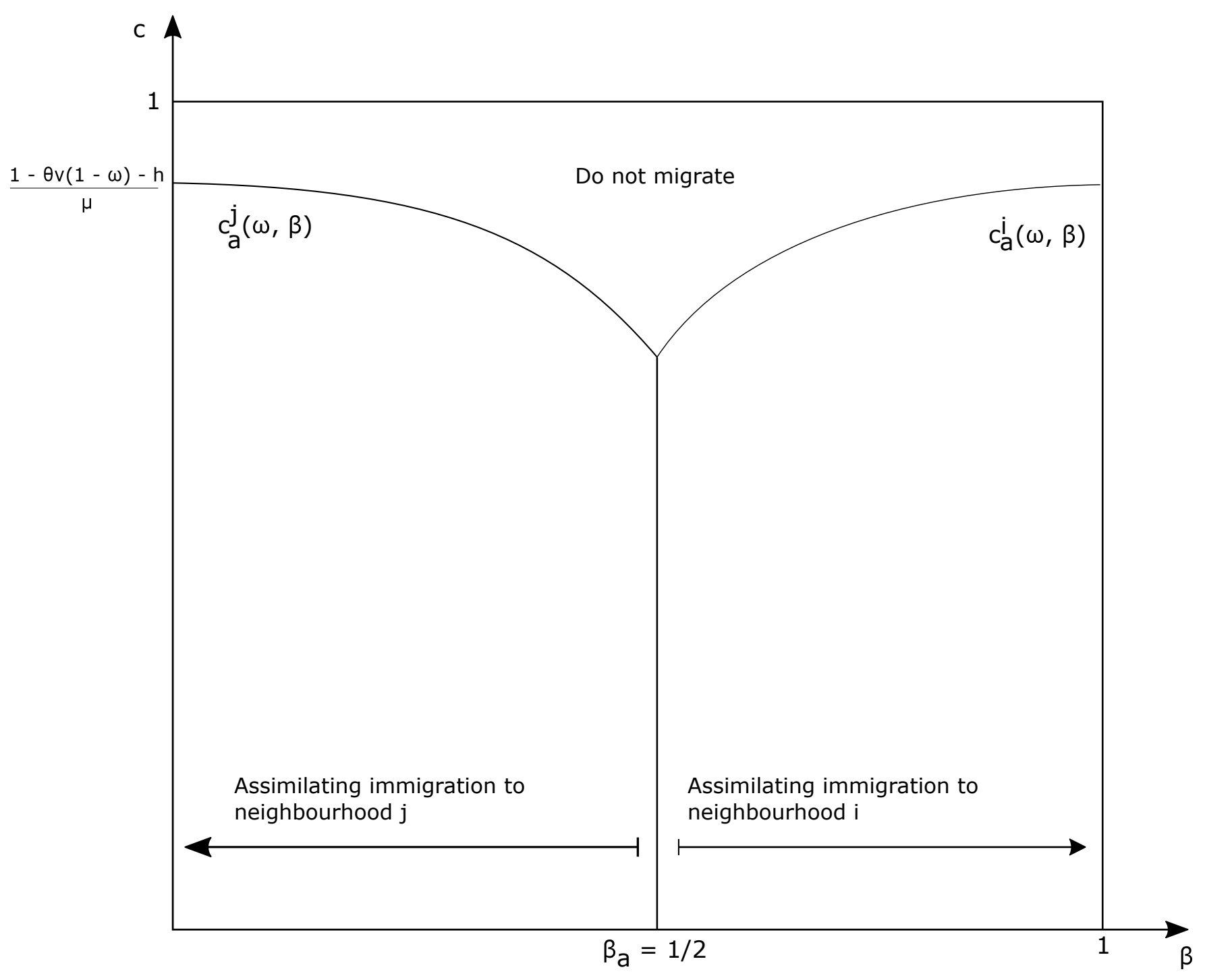


MONTEIRO Cultural Assimilation: Learning and Sorting

Figure 4: Mixed equilibrium; $\beta>1 / 2, m^{j} \geq m^{i}$ and $h<m^{i} m^{j} /\left(m^{i}+m^{j}+2 m^{i} m^{j}\right)$

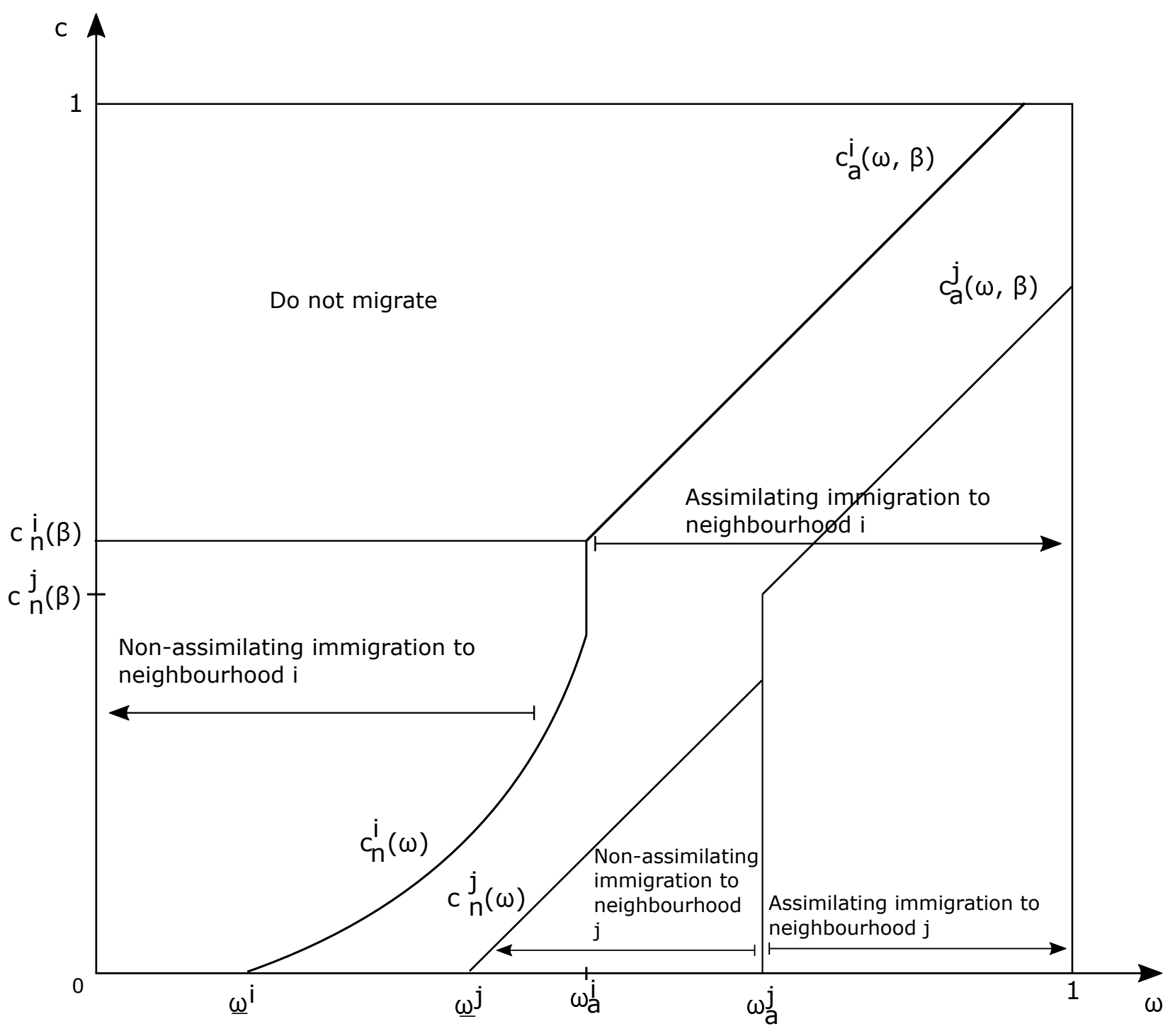


Similarly, non-assimilating immigration to neighbourhood $i$ must satisfy the condition that $c \leq$ $c_{n}^{i}(\beta), \omega<\omega_{a}^{i}$ and $\beta>\beta_{n}(c)$. Substitute $\beta_{n}(c)$ into $c_{a}^{i}(\omega, \beta)$ and implicitly define $c_{n}^{i}(\omega)$ as

$$
c_{n}^{i}(\omega)+\frac{h}{\mu}\left(\frac{m^{i} /\left(1+m^{i}\right)}{m^{j} /\left(1+m^{j}\right)-2 \mu c_{n}^{i}(\omega)}\right)=\frac{1-\theta v(1-\omega)-h}{\mu}
$$

Equations (15) and (16) are depicted in figure 4. This information is sufficient to identify the non-assimilating immigrant groups in both neighbourhoods.

\subsection{Equilibria}

Two equilibrium states exist for $m^{i} \in[0, \infty)$ : a sorting and mixed equilibrium. One of the two states will emerge given levels of $h$. Each equilibrium has certain qualitative features. For simplicity of exposition let the the level of $\omega$ at which $c_{n}^{i}(\omega)=0$ and $c_{n}^{j}(\omega)=0$ be defined respectively as

$$
\begin{gathered}
\underline{\omega}^{i} \equiv 1-\frac{1-h\left(1+m^{i}\left(1+m^{j}\right) / m^{j}\left(1+m^{i}\right)\right)}{\theta v} \\
\underline{\omega}^{j} \equiv 1-\frac{1-h\left(1+m^{j}\left(1+m^{i}\right) / m^{i}\left(1+m^{j}\right)\right)}{\theta v}
\end{gathered}
$$

In a mixed equilibrium, immigration is mixed within neighbourhoods. That is, there is positive levels of immigration in both neighbourhoods and there is assimilating and non-assimilating immigration present. The mixed equilibrium outcome in neighbourhood $i=A, B$ is given by

$$
\begin{gathered}
a^{i}=L \int_{\omega_{a}^{i}}^{1} \int_{1 / 2}^{1} F\left(c_{a}^{i}(\omega, \beta)\right) d B(\beta) d W(\omega) \\
m^{i}-a^{i}=L W\left(\omega_{a}^{i}\right) \int_{\frac{h\left(1+m^{i}\right)}{m^{i}}}^{1} \max \left\{0, F\left(c_{n}^{i}(\beta)\right)-F\left(c\left(\beta_{n}\right)\right)\right\} d B(\beta) \\
\text { and } \omega_{a}^{i}>\underline{\omega}^{i} .
\end{gathered}
$$

The left-hand side of (17) - (18) is the actual number of assimilating immigrants $\left(a^{i}\right)$ and nonassimilating immigrants $\left(m^{i}-a^{i}\right)$, respectively. These are equated to their respective expected values. Similarly, the outcomes in a mixed equilibrium for neighbourhood $j=A, B$ where $j \neq i$ is given by

$$
\begin{gathered}
a^{j}=L \int_{\omega_{a}^{j}}^{1} \int_{0}^{1 / 2} F\left(c_{a}^{j}(\omega, \beta)\right) d B(\beta) d W(\omega) \\
m^{j}-a^{j}=L\left(W\left(\omega_{a}^{j}\right)-W\left(\underline{\omega}^{j}\right)\right) \int_{0}^{\frac{m^{j}\left(1+m^{i}\right)}{m^{i}+m^{j}+2 m^{i} m}} F\left(c\left(\beta_{n}\right)\right) d B(\beta) \\
\text { and } \omega_{a}^{j}>\underline{\omega}^{j} .
\end{gathered}
$$

The left-hand side of (19) - (20) is the actual number of assimilating immigrants $\left(a^{j}\right)$ and nonassimilating immigrants $\left(m^{j}-a^{j}\right)$, respectively. These are equated to their expected values. 
MONTEIRO Cultural Assimilation: Learning and Sorting

Table 1: Mixed or sorting equilibrium outcomes given $h$.

\begin{tabular}{ccccc}
\hline \hline$\omega_{a}^{i}$ & $\omega_{a}^{j}$ & & & Outcome \\
\cline { 4 - 5 }$\geq \underline{\omega}^{i}$ & $\underline{\omega}^{j}$ & & Rich & Sorting \\
$\geq \underline{\omega}^{i}$ & $>\underline{\omega}^{j}$ & & Poor & Mixed \\
\hline \hline
\end{tabular}

Total immigration is $m=m^{i}+m^{j}$ and total assimilating immigration is $a=a^{i}+a^{j}$. Note that the mixed equilibrium conforms to the corner equilibrium in Konya (2007) when $\omega_{a}^{j}=\underline{\omega}^{j}$ and $c_{n}^{i}(\beta)=0$ giving the special case in which $m^{i}=m^{j}$.

In a sorting equilibrium there is mixed immigration in one neighbourhood and only assimilating immigration in the other. The sorting equilibrium is given by equations (17) - (20) and

$$
\omega_{a}^{i} \geq \underline{\omega}^{i} \text { and } \omega_{a}^{j}=\underline{\omega}^{j}
$$

for all $i, j=A, B$ and $j \neq i$. Immigration to neighbourhood $i$ is mixed but only assimilating in neighbourhood $j$. Table 1 further summarizes the outcomes given $h$.

Although the equilibria are not unique, they are stable under very reasonable conditions. Stability in the two neighbourhood case must satisfy the following condition

$$
\left\|J\left(m^{i}, m^{j}\right)\right\|<1
$$

on $D=\left\{\left(m^{i}, m^{j}\right) \mid m^{i}, m^{j}>0\right\}$ where $J\left(m^{i}, m^{j}\right)$ is the Jacobian matrix of first-order derivatives of the equation system describing the mixed equilibrium outcomes in neighbourhoods $i$ and $j$.

$$
\begin{aligned}
m^{i}=L & \int_{\omega_{a}^{i}}^{1} \int_{1 / 2}^{1} F\left(c_{a}^{i}(\omega, \beta)\right) d B(\beta) d W(\omega) \\
& +W\left(\omega_{a}^{i}\right)^{2} \int_{\frac{h\left(1+m^{i}\right)}{m^{i}}}^{1} \max \left\{0, F\left(c_{n}^{i}(\beta)\right)-F\left(c\left(\beta_{n}\right)\right)\right\} d B(\beta) \equiv \Gamma^{i}\left(m^{i}, m^{j}\right) \\
m^{j}=L & \int_{\omega_{a}^{j}}^{1} \int_{0}^{1 / 2} F\left(c_{a}^{j}(\omega, \beta)\right) d B(\beta) d W(\omega) \\
& +L\left(W\left(\omega_{a}^{j}\right)-W\left(\underline{\omega}^{j}\right)\right)^{2} \int_{0}^{\frac{m^{j}\left(1+m^{i}\right)}{m^{i}+m^{j}+2 m^{i} m^{j}}} F\left(c\left(\beta_{n}\right)\right) d B(\beta) \equiv \Gamma^{j}\left(m^{i}, m^{j}\right) \\
\omega_{a}^{i} \geq & \underline{\omega}^{i} \text { and } \omega_{a}^{j}>\underline{\omega}^{j} .
\end{aligned}
$$

The first-order derivatives are provided in the comparative statics section of the appendix. Stability of the mixed equilibrium system requires that the eigenvalues of $J\left(m^{i}, m^{j}\right)$ are signed as follows

$$
\frac{\partial \Gamma^{i}}{\partial m^{i}}<0 \text { and } \frac{\partial \Gamma^{j}}{\partial m^{j}}<0
$$


The stability conditions also imply that $\partial \Gamma^{j} / \partial m^{i}>0$. The stability of a sorting equilibrium must also satisfy condition (24), although $\partial \Gamma^{j} / \partial m^{j}<0$ and $\partial \Gamma^{j} / \partial m^{i}>0$ hold without assumption. The first-order derivatives in the sorting case are also provided in the comparative statics section of the appendix.

Finally, the emergence of either equilibrium type is dependent on the level of $h$ as proposed in column 4 of table 1. Proposition 1 below proves the existence of a level of $h$ that switches the equilibrium outcome from a mixed to a sorting.

Proposition 1. There exists an $\bar{h}$, such that, for $h<\bar{h}$ the equilibrium is sorting, and mixed for $h>\bar{h}$.

Proof. Let $\bar{m}^{j}$ be the solution to $\omega_{a}^{j}>\underline{\omega}^{j}$ in a mixed equilibrium outcome. Similarly, the sorting outcome is given by substituting $\omega_{a}^{j}=\underline{\omega}^{j}$ into the right-hand side of equation (23). Equating the former $\bar{m}^{j}$ with the latter gives the following implicit function defining $\bar{h}$ by

$$
\frac{\bar{h}}{1-2 \bar{h}-\bar{h} / m^{i}(L, h, \mu, \theta)}=L \int_{\underline{\omega}^{j}}^{1} \int_{0}^{1 / 2} F\left(c_{a}^{j}(\omega, \beta)\right) d B(\beta) d W(\omega) .
$$

Note that both sides of the equation include $m^{i}(L, h, \mu, \theta)$, as defined by equation 22 , which is also a function of $h$. Let $\phi\left(m^{i}(L, \bar{h}, \mu, \theta), m^{j}(L, \bar{h}, \mu, \theta), L, \bar{h}, \mu, \theta\right)=0$ be the difference between the left-hand and right-hand side of equation (25). The comparative statics in the appendix show that $\partial m^{i} / \partial h<0$, as such, the left-hand side of equation (25) is monotonically increasing in $h \in[0,1)$, but is discontinuous at $h=1$, at which point it becomes zero. The right-hand side is continuous and monotonically decreasing in $h \in[0,1]$, and zero at $h=1$. At $h=0$ the right-hand side is larger than the left-hand side. And, at $h=1$ the left-hand side and right-hand side are both zero and equal. Figure 5 shows clearly the existence of $\bar{h}$. The left-hand hand side of equation (25) is clearly shown by the upward sloping linear function with a discontinuity at $h=1$. Similarly, the right-hand side of equation (25) and $m^{i}(L, h, \mu, \theta)$ is also depicted as the downward sloping functions, where $m^{j} \geq m^{i}$. The three equations depicted in figure 5 are not linear in $h$, instead they are concave/convex at different levels of $h \in(0,1)$; linearity is imposed for the sake of simplicity in exposition. Since the functions are monotonically increasing/decreasing in $h \in(0,1)$, linearity suffices to depict the existence of $\bar{h}$. The threshold level of $h$ at which point the equilibrium changes from the mixed to the sorting case is indicated by

$$
\bar{h}=\frac{m^{i} m^{j}}{m^{i}+m^{j}+2 m^{i} m^{j}} .
$$

Consistent with the graphical depiction is the result that a higher $h$ is associated with a lower level of immigration in both neighbourhoods. Moreover, changes in the parameter set $\{L, h, \mu, \theta\}$ will have implications for the level of $\bar{h}$ and, in turn, on the equilibrium outcome. 
MONTEIRO Cultural Assimilation: Learning and Sorting

Figure 5: Existence of $\bar{h} ; m^{j} \geq m^{i}$

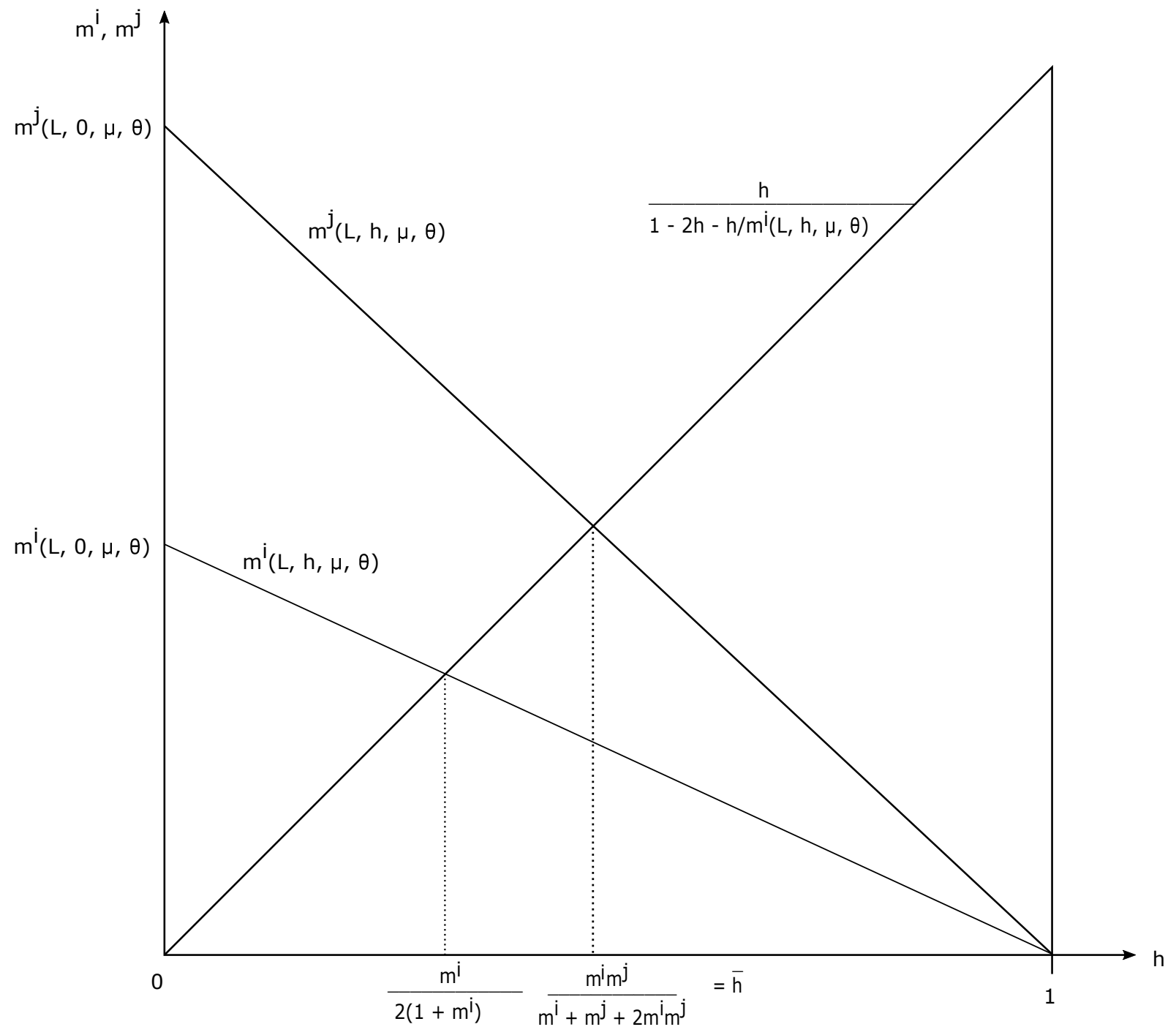


Under proposition 1, a richer source country does not necessarily imply that all immigration will be assimilating, as was the case in Konya (2007). Instead I find that one of the two neighbourhoods in the North continues to receive the non-assimilating type even if the source country is rich.

The intuition behind this result is best understood as a productivity, network and communication effect. Efficiency of matches in the South has an impact on the level of immigration. As the South gets poorer there is more migration from the South because matches in the North become relatively more productive. Incurring the migration costs and foregoing the matches that could have been made in the South (the opportunity cost), the potential migrant finds these costs are small relative to the gains from matches in the North. This represents a productivity effect increasing the total number of immigrants from poorer source countries. Non-assimilating immigrants experience a debilitating communication effect if their pre-immigration experience with the local culture is not large. Some of them will have large enough pre-immigration experience with the local culture to enter the assimilating group. The communication effect determines the composition of immigration within neighbourhoods. Only if the communication effect is larger than the productivity effect will all immigration become assimilating.

Given the productivity and communication effects, networks function to induce new immigrants to choose neighbourhoods where they have more family/friends. If the productivity effect is large enough then total immigration is increasing. But based on the relative size of pre-existing networks new immigrants will have a general preference for some neighbourhoods over others.

\section{Empirical Results}

This section will test the implications of the theoretical model. There are four subsections: a description of the data, a statement of the model's theoretical predictions, the empirical model identification strategy, estimation results, and further insights.

\subsection{Data}

The data used in this study is from the Longitudinal Survey of Immigrants in Canada (LSIC). The survey is a three wave study conducted on new immigrants and refugees to Canada, atleast 15 years of age, in the period October 2000 and September 2001 (approximately 65,000 new immigrants). The survey excludes applications for immigration or asylum made within Canada. The cohort of immigrants captured in the survey were subject to the non-discriminatory character of the 1976 Immigration Act (Bodvarsson \& Berg, 2013) and were admitted prior to the 2002 Immigration and Refugee Protection Act. The first wave is collected between April 2001 and March 2002, six months after arrival (12,040 immigrants of the 65,000 were recorded). A second wave of data is collected on this same group of individuals six months later, between December 2002 and November 2003 (9,500 immigrants were re-recorded). A final wave is 


\section{MONTEIRO Cultural Assimilation: Learning and Sorting}

conducted one year later, between November 2004 and October 2005 (7,715 immigrants were re-re-recorded) (Haan, 2012). The attrition rate is $21.1 \%$ and $18.8 \%$ in waves 2 and 3. Attrition is especially important in a study such as this because those immigrants that were lost from the sampling between waves 1-2 and waves 2-3 have important information about their level of assimilation or non-assimilation.

The focus of this study is new immigrants with little pre-immigration experience and low levels of exposure to Canadian culture. The subsample used in this study are immigrants whose mother tongue is non-English and do not reside in Quebec. This subsample is used throughout unless mentioned otherwise.

Immigrants may become missing from subsequent waves for various reasons: change in address and no follow-up contact information was provided, become deceased, or return to the home country. The reason for becoming missing from subsequent waves is not recorded. This becomes an issue because immigrants that returned to their home country due to difficulties in the assimilation process will bias the final results of the model. Only the remaining successful assimilates will be recorded and immigrant assimilation is overestimated. Characteristics of the returnees may be nonrandom and the bias will be exacerbated.

There are two advantages to using the LSIC over cross-sections such as in Konya (2007) and Lazear (1999). Firstly, the LSIC has a large number of pre-immigration characteristics that are unavailable in the Census. Pre- and post-immigration formal and informal learning of the local culture allows for controlling the learning effect to isolate the sorting effect in the model. Secondly, the LSIC allows for the construction of a valid proxy for living and working in an ethnic enclave. The disadvantage with the LSIC is that it only includes a single cohort of immigrants from 2000-2001. However, this is not a major disadvantage given the trade-off. The LSIC has depth on a single cohort of immigrants, but the Census has shallow information on many cohorts. Since the pre-immigration variables and learning of the local culture is shown to be important (described further in the results section below) and must be included in the model, the LSIC provides new important information that is unavailable in a study that solely uses the Census.

\subsection{Model predictions}

An empirical test of the relationships implied by the model requires the following definition for assimilating immigration $\rho^{j}=a^{j} / \mathrm{m}^{j}$ in neighbourhood $j=A, B$. The measure of assimilating immigration $\rho$ measures within neighbourhood composition of immigration in the North. Since the $j^{\text {th }}$ neighbourhood receives no non-assimilating immigration in the sorting equilibrium, the predicted outcomes of the model are summarized simply by

$$
\rho^{j}= \begin{cases}1 & \text { if } h \geq \bar{h}, \\ \rho^{j}\left(m^{j}(h, \theta, \mu, L), m^{i}(h, \theta, \mu, L), h, \theta, \mu\right) & \text { if } h<\bar{h}\end{cases}
$$


Review of Economic Analysis 13 (2021) 115-156

where $\bar{h}$ is defined by $\phi\left(m^{i}(\bar{h}, \theta, \mu, L), m^{j}(\bar{h}, \theta, \mu, L), \bar{h}, \theta, \mu, L\right)$ from equation (25), $i, j=A, B$ and $i \neq j$. The parameters $(L, h, \mu, \theta)$ impact the type of equilibrium through a threshold, direct or indirect effect. The threshold effect determines the type of equilbrium in neighbourhood $j$ through changes in $\phi($.$) . Changes in the parameters have a direct impact on the composition of$ immigration within the mixed equilibrium through changes in $\rho^{j}($.). The indirect effects of the model are associated with changes in the composition through the size of immigration. Since there are two neighbourhoods to consider, the parameters effect the composition of immigration through $m^{i}$.) and $m^{j}($.). Table 2 summarizes the various effects.

Table 2: The effect of parameters on likelihood of assimilation $\rho^{j}$

\begin{tabular}{ccccc}
\hline \hline \multirow{2}{*}{ Parameter } & \multicolumn{4}{c}{$\rho^{j}\left(m^{j}(h, \theta, \mu, L), m^{i}(h, \theta, \mu, L), h, \theta, \mu\right)$} \\
\cline { 2 - 5 } & Threshold & Direct & Indirect $m^{j}(h, \theta, \mu, L)$ & Indirect $m^{i}(h, \theta, \mu, L)$ \\
\hline \multirow{2}{*}{$L$} & - & 0 & $?$ & $?$ \\
$h$ & + & $?$ & $?$ & $?$ \\
$\mu$ & + & - & $?$ & $?$ \\
$\theta$ & $?$ & $?$ & $?$ & $?$ \\
\hline \hline
\end{tabular}

The full effect of the parameters on the composition of immigration are ambiguous, the empirics cannot be used as a test of the model. On the other hand, although most immigrant receiving countries will restrict immigration through a quota or points system, the total size of immigration is typically exogeneously fixed at $m=m^{i}+m^{j}$. However, the model predictions function through the relative size of immigration across neighbourhoods, which is determined by individual preferences, some source country characteristics, and not the immigration authorities. Let $\zeta \equiv m^{i} /\left(m^{i}+m^{j}\right)$ measure the distribution of immigrants across neighbourhoods in the North. The effects of the parameters on the distribution of immigration are also estimated and discussed in the further insights section. ${ }^{4}$

\subsection{Empirical model}

Exposure to co-ethnics is the effective channel through which source country richness impacts assimilating immigration in the model. Since sorting amplifies the effects of exposure I expect source country richness' effect on assimilating immigration to function through the sorting variable.

Identifying the exposure channel through which source country richness affects assimilating

${ }^{4}$ See the appendix for further discussion on the direct, indirect and threshold effects. 


\section{MONTEIRO Cultural Assimilation: Learning and Sorting}

immigration requires a regression framework that controls for alternate channels, such as, selection from within the source country, exposure within the household and immigration policy in the host country. Similarly, factors such as pre-immigration experience with the local culture, individual learning costs and other unobservable characteristics of the immigrant will probably affect the sorting and learning decision simultaneously. Each of these are discussed and treated appropriately in the following sections.

Sorting increases exposure to co-ethnics, through which source country richness affects assimilation rates. A proxy for the sorting variable is included in the model which intentionally over-adjusts (or over-controls) for the exposure channel. This addition to the model may be used to determine if sorting is indeed the effect being estimated in the exposure channel between source country richness and assimilation rates.

The estimation equation is a regression model to determine the effect of exogenous timevarying characteristics $\left(X_{i s t}\right)$, exogenous time-invariant variables $\left(X_{i s}\right)$, investment into language accumulation $\left(\iota_{i s t}\right)$, living/working in an ethnic enclave in CMA/CA of arrival $\left(\zeta_{i s}\right)$, and source country richness $\left(h_{i s}\right)$ on the immigrant's decision to assimilate overtime. The $X_{i s t}$ and $X_{i s}$ factors are treated as exogenous; they include demographic and economic information on immigrants as well as pre-immigration experience with the local culture and friend/family networks. The time-invariant variable $X_{i s}$ includes contextual variables, such as, source country's linguistic distance, population, geographic distance, and a measure of the share of co-ethnics in Canada at the time of arrival $m_{i s}$. The variable $\iota_{i s t}$ includes formal and informal investment into language accumulation. Living/working in an ethnic enclave $\zeta_{i s}$ is measured by choosing to initially live in a CMA/CA where co-ethnics are clustered and working in an organization that is predominately co-ethnic; this variable is endogenous. The assimilation variable $\rho_{\text {ist }}$ is proxied by language proficiency, where immigrant $i$ is from source country $s$ and measured in period $t$.

The three waves of the survey are treated as a panel. The benchmark regression model to estimate is simply the effect of source country richness $\left(h_{i s}\right)$ on assimilating immigration $\left(\rho_{i s t}\right)$.

$$
\rho_{i s t}=\gamma_{0}+\gamma_{1} h_{i s}+\gamma_{4} X_{i s}+\gamma_{5} X_{i s t}+\eta_{i}+\epsilon_{i s t} .
$$

The estimated effect of $h_{i s}$ on $\rho_{i s t}$ is $\widehat{\gamma}_{1}$. This estimate captures exposure to co-ethnicity and learning simultaneously. The next equation will control for investment in formal and/or informal learning $\iota_{i s t}$,

$$
\rho_{i s t}=\gamma_{0}+\gamma_{1} h_{i s}+\gamma_{3} \iota_{i s t}+\gamma_{4} X_{i s}+\gamma_{5} X_{i s t}+\eta_{i}+\epsilon_{i s t} .
$$

The estimated effect of $h_{i s}$ on $\rho_{i s t}$ through the hypothesized exposure channel is $\widehat{\gamma}_{1}$. Moreover, exposure to co-ethnics occurs within or across neighbourhoods, and allows for non-random location choices. The time-varying and -invariant variables in the model control for alternate channels and confounding relationships. If the observed relationship between source country 
richness and assimilating immigration is through the exposure channel then by including the distribution measure into the model over-adjusts for the sorting effect. This procedure intentionally controls for the sorting effect in $\widehat{\gamma}_{1}$ that this study is trying to identify. This over-adjustment will confirm whether sorting is the only effect being captured by the estimated $\widehat{\gamma}_{1}$. The following regression model includes the measure for distribution of immigrants across neighbourhoods $\left(\zeta_{i s}\right)$,

$$
\rho_{i s t}=\gamma_{0}+\gamma_{1} h_{i s}+\gamma_{2} \zeta_{i s}+\gamma_{3} \iota_{i s t}+\gamma_{4} X_{i s}+\gamma_{5} X_{i s t}+\eta_{i}+\epsilon_{i s t} .
$$

The coefficient estimate of language investment is biased because of simultaneity; non-assimilating immigrants are more likely to invest into learning the local culture. There is correlation between $\iota_{i s t}$ and the individual-level error component $\eta_{i}$, implying that the coefficient on language investment will not be signed precisely without a fixed effects estimator. That being said, causal effects of investment are not the purpose of this paper.

I expect $\eta_{i}$ to capture factors that inhibit or ease the selection into formal language programs not currently captured in (28), such as distance to nearest ESL course, time cost, program costs, and ability to learn new languages. Finally, the time-invariant variable $\zeta_{i s}$ is endogenous because new immigrants with low English speaking skills will choose smaller communication frictions by locating in ethnic enclaves where access to ethnic goods is easy and learning the local culture can happen in a low cost environment.

Immigrants that live and work in an ethnic enclave are identified using data from the Longitudinal Survey of Immigrants in Canada: wave 1 and the 2001 Canadian Census of Population. The LSIC was used to provide information on whether the immigrant worked in an organization that predominately spoke a language other than English or French, in wave 1. The Census was used to identify whether a given ethnic group is over-represented in a census tract (CT) relative to the CMA/CA in which it is located. If more than 50\% of CT's are over-representative of the ethnic group then the $\mathrm{CMA} / \mathrm{CA}$ is considered to be clustering co-ethnics. Immigrants in the LSIC are identified as living/working in an ethnic enclave if they work in an organization predominately speaking a language other than English or French in wave 1, and if they belong to an ethnic group that lived in a CMA/CA of first arrival that clustered co-ethnics as determined by the Census.

A standard set of controls are used throughout. The controls that enter the model are based on previous studies that have shown the specific variable to be an important determinant of English speaking proficiency. The variables used in this study are described in table 8 of the appendix. Given that the language proficiency variable is subjectively determined there is potential response error arising from lack of a stable benchmark. That is, respondents in the LSIC may report decreasing language proficiency across waves. Whether the decreases in language proficiency are actually due to worsening language skills or due to a lack of a benchmark is difficult to determine. This is problematic because, in the estimation strategy described above, I 


\section{MONTEIRO Cultural Assimilation: Learning and Sorting}

may wrongly categorize a respondent to having worsening language proficiency when the problem was simply a response error. This error will underestimate assimilation levels. The extent of the measurement error is described in table 3 below

Table 3: Measurement error in English speaking proficiency across waves.

\begin{tabular}{cccccc}
\hline \hline & \multicolumn{2}{c}{ Wave 1 - } & & \multicolumn{2}{c}{ Wave 2 - 3 } \\
\cline { 2 - 3 } \cline { 5 - 5 } \cline { 5 - 5 } & Frequency & Percentage & & Frequency & Percentage \\
\hline \multirow{2}{*}{ Worse } & 14,798 & $15 \%$ & & 19,286 & $20 \%$ \\
Better & 28,379 & $29 \%$ & & 21,812 & $22 \%$ \\
Same & 53,870 & $56 \%$ & & 55,949 & $58 \%$ \\
\hline Total & 97,047 & $100 \%$ & & 97,047 & $100 \%$ \\
\hline \hline
\end{tabular}

Note: The summary statistics produced in this table uses the raw categorical five-level English speaking proficiency variable to be as accurate as possible.

At most $15 \%$ of new immigrants that provided responses in waves 1 and 2, and $20 \%$ in waves 2 and 3, could be attributed to the measurement error. Since this error would underestimate assimilation levels, the results of this study may be interpreted as a lower bound. For the remainder of the paper, the five-level categorical English speaking variable will be collapsed into a dummy variable which would minimize the influence of the measurement error. But if the error is non-random then the bias will persist. It is expected that the measurement error is more likely to occur among refugees, those with low English speaking proficiency, those who are less educated, and have less experience with the English language. Table 4 shows there is no systematic response in worsening English speaking proficiency between refugees and nonrefugees, and between those with less than a high school education and those with at least a high school education. Moreover, those with pre-immigration experience with the English language are more likely to respond with worsening English speaking skills than those without; either because they are understating their knowledge of the English language or they discover, after immigration, that the quality of their pre-immigration experience is low.

On the other hand, those with lower English speaking proficiency in wave $t$ are more likely to report worsening English speaking proficiency in wave $t+1$ compared to those with higher proficiency in wave $t$. This evidence indicates there will be some bias associated with measurement error. The measurement error in the dependent variable will bias coefficient estimates of the time-invariant variables in the model. 
Table 4: Worsening of English speaking proficiency across waves $t$ and $t+1$ by refugee status, English speaking proficiency, education, and formal pre-immigration experience with the English language.

\begin{tabular}{|c|c|c|c|c|c|c|c|c|}
\hline & \multicolumn{2}{|c|}{ Refugee } & \multicolumn{2}{|c|}{ Low proficiency } & \multicolumn{2}{|c|}{ Less than high school } & \multicolumn{2}{|c|}{ Pre-immigration experience } \\
\hline & Yes & No & Yes & No & Yes & No & Yes & No \\
\hline & \multicolumn{8}{|c|}{ Waves 1 - 2} \\
\hline Worse & 14.76 & 15.28 & 29.39 & 9.62 & 13.86 & 15.49 & 17.74 & 7.92 \\
\hline Better & 46.61 & 28.20 & 21.80 & 32.21 & 35.32 & 28.16 & 29.45 & 28.67 \\
\hline \multirow[t]{2}{*}{ Same } & 38.63 & 56.53 & 48.81 & 58.17 & 50.82 & 56.34 & 52.81 & 63.41 \\
\hline & \multicolumn{8}{|c|}{ Waves 2 - 3} \\
\hline Worse & 22.96 & 19.69 & 39.09 & 12.57 & 20.75 & 19.72 & 21.42 & 15.41 \\
\hline Better & 34.33 & 21.76 & 15.30 & 25.20 & 22.26 & 22.51 & 23.67 & 19.01 \\
\hline Same & 42.71 & 58.55 & 45.61 & 62.23 & 56.99 & 57.77 & 54.91 & 65.58 \\
\hline
\end{tabular}

Note 1: The summary statistics produced in this table uses the raw categorical five-level English speaking proficiency variable.

Note 2: Low proficiency is measured by speaking English fairly well, poorly and not at all in wave $t+1$. Note 3: Less than high school is measured by highest level of formal education attained outside Canada in wave $t+1$.

\subsection{Results}

Firstly, I estimate the benchmark model of equation (28). The probit estimates are presented below in the first column of table 5. The effect of source country richness on assimilating immigration is measured by the coefficient on RGDP per capita. As expected, source country richness has a significant positive effect on assimilating immigration. But this estimate is capturing both, the channel of exposure to co-ethnicity and learning.

The second equation (29) controls for learning by including the set of formal/informal investment variables. Now the coefficient estimate on RGDP per capita is insignificant as shown in column 2 of table 5. This would say that exposure to co-ethnicity is not a part of this channel. However, OLS estimates are significant (see column 3 of table 10).

The third column of table 5 includes the network variable $(\beta)$ into the model. This variable controls for individual preference in neighbourhood choice. Networks are proxied by whether the immigrant had family/friends in their current area (or nearby area) of residence and chose the area because family/friends live there. The effect of this variable on assimilating immigration is negative but insignificant. The source country richness coefficient also remains insignificant.

The fourth column includes the ethnic enclave variable over-adjusting for the implied sorting effect. Including this variable in the model changes the coefficient estimate of RGDP per capita 


\section{MONTEIRO Cultural Assimilation: Learning and Sorting}

and makes it significant. This indicates that there is an alternate channel through which source country richness affects assimilation rates.

The ethnic enclave variable $\zeta$ is endogenous because immigrants with low English speaking skills are more likely to choose to live and work within the enclave. Additionally, the ethnic enclave variable is interacting with source country richness. I instrument the ethnic enclave variable with housing costs in CMA/CA of arrival from the 2001 Canadian Census of Population. Housing costs are significantly and positively correlated with living and working in an ethnic enclave because new immigrants will choose to live in the low cost ethnic enclave if the CMA/CA has high average cost of housing. Table 6 provides a sorting regression where the coefficient estimate of housing costs in CMA/CA of arrival is significant and positive. Also, housing costs in CMA/CA of arrival has no observed association with assimilation rates. The fifth column instruments the ethnic enclave variable with housing costs in CMA/CA of arrival. The estimated effect of living and working in an ethnic enclave becomes larger. The coefficient effect of source country richness is still significant, implying the presence of an alternate channel. The further insights section discusses a possible alternate pathway.

Investment $\iota$ is measured by further learning of English in Canada. Further learning is differentiated by formal and informal means. Formal and informal investment have a significant effect on assimilating immigration. The sign of formal and informal learning is negative because of simultaneity.

A dummy variable for pre-immigration formal and informal learning of English proxies for $\omega$ in the theoretical model. Pre-immigration learning of English enters significantly and positive. This is expected since individuals with higher levels of pre-immigration experience with the local culture are more likely to enter the assimilating group if their experience is large, or have a low investment cost to learning the local culture.

The restrictions implied by the immigration policy in place at the time of arrival are proxied by ethnic share in Canada at the start of the survey $(m)$. The coefficient estimate of this variable on assimilating immigration is positive but insignificant.

Finally, the coefficients for population levels $(L)$ relative to Canada, linguistic distance $(\theta)$ and geographic distance $(\mu)$ of the capital city in country of birth to Ottawa are estimated. The effect of source country population on English speaking proficiency is negative. Geographic distance and cultural differences have no significant effect on assimilating immigration.

In table 10 of the appendix I estimate the benchmark model of equation (28) by OLS. The fourth column of table 10 subsets the data to those source countries that scored "low" or "very low" on the English proficiency index (EF EPI, 2017) ${ }^{5}$. There is much less variation in RGDP per capita and the countries were predominately low income. As expected the coefficient estimate on RGDP per capita is insignificant but still positive.

${ }^{5}$ Many African and Eastern European countries were not surveyed for the index. These were also omitted in the sub-sample. 
Review of Economic Analysis 13 (2021) 115-156

Refugees in the LSIC represent $15 \%$ of the sample. Although this is considerable, excluding this group from the estimation model makes no difference to the estimated coefficients on language investment and living/working in an ethnic enclave. Also, the survey weighting accounts for the oversampling.

Table 5: Probit estimation results of English speaking proficiency: households whose mother tongue is not English and do not reside in Quebec.

\begin{tabular}{|c|c|c|c|c|c|}
\hline $\begin{array}{l}\text { Dependent variable: English speaking } \\
\text { proficiency }\end{array}$ & Pooled & Pooled & Pooled & Pooled & Pooled IV \\
\hline \multirow[t]{2}{*}{ RGDP per capita $(h)$} & $0.156^{* *}$ & 0.119 & 0.116 & $0.129^{*}$ & $0.150^{* *}$ \\
\hline & $(0.0555)$ & $(0.0614)$ & $(0.0614)$ & $(0.0630)$ & $(0.0569)$ \\
\hline \multirow[t]{2}{*}{ Network $(\beta)$} & & & -0.0827 & -0.0716 & 0.0363 \\
\hline & & & $(0.0572)$ & $(0.0573)$ & $(0.0574)$ \\
\hline \multirow[t]{2}{*}{ Ethnic enclave $(\zeta)$} & & & & $-0.368^{* * *}$ & $-2.588^{* * *}$ \\
\hline & & & & $(0.0602)$ & $(0.384)$ \\
\hline \multirow[t]{2}{*}{ Informal investment ( $\iota)$} & & $-0.272^{* * *}$ & $-0.271^{* * *}$ & $-0.273^{* * *}$ & $-0.144^{*}$ \\
\hline & & $(0.0434)$ & $(0.0435)$ & $(0.0431)$ & $(0.0641)$ \\
\hline \multirow[t]{2}{*}{ Formal investment $(\iota)$} & & $-0.241^{* * *}$ & $-0.242^{* * *}$ & $-0.248^{* * *}$ & $-0.203^{* * *}$ \\
\hline & & $(0.0482)$ & $(0.0482)$ & $(0.0483)$ & $(0.0542)$ \\
\hline \multirow[t]{2}{*}{ Formal pre-immigration experience $(\omega)$} & & $0.618^{* * *}$ & $0.617^{* * *}$ & $0.616^{* * *}$ & $0.367^{* *}$ \\
\hline & & $(0.0510)$ & $(0.0510)$ & $(0.0512)$ & $(0.119)$ \\
\hline \multirow{2}{*}{$\begin{array}{l}\text { Informal pre-immigration experience } \\
(\omega)\end{array}$} & & $0.360^{* * *}$ & $0.358^{* * *}$ & $0.348^{* * *}$ & $0.174^{*}$ \\
\hline & & $(0.0501)$ & $(0.0501)$ & $(0.0504)$ & $(0.0837)$ \\
\hline \multirow[t]{2}{*}{ Share of co-ethnic group in Canada $(\mathrm{m})$} & 1.709 & 0.0104 & 0.0522 & 0.553 & $3.200^{* *}$ \\
\hline & $(1.038)$ & $(1.037)$ & $(1.036)$ & $(1.042)$ & $(1.074)$ \\
\hline \multirow[t]{2}{*}{ Population level $(L)$} & $-0.0257^{* * *}$ & $-0.0168^{* *}$ & $-0.0169^{* *}$ & $-0.0183^{* *}$ & $-0.0197^{* * *}$ \\
\hline & $(0.00600)$ & $(0.00612)$ & $(0.00611)$ & $(0.00620)$ & $(0.00586)$ \\
\hline \multirow[t]{2}{*}{ Geographic distance $(\mu)$} & 0.00163 & $-0.0242^{*}$ & -0.0237 & -0.0231 & -0.00563 \\
\hline & $(0.0118)$ & $(0.0123)$ & $(0.0123)$ & $(0.0123)$ & $(0.0116)$ \\
\hline \multirow[t]{2}{*}{ Linguistic distance $(\theta)$} & -0.0526 & -0.00734 & -0.0102 & 0.00866 & 0.0378 \\
\hline & $(0.219)$ & $(0.222)$ & $(0.222)$ & $(0.222)$ & $(0.176)$ \\
\hline \multirow[t]{2}{*}{ Months since arrival } & $-0.0399^{*}$ & -0.0363 & -0.0366 & -0.0373 & -0.0290 \\
\hline & $(0.0196)$ & $(0.0205)$ & $(0.0205)$ & $(0.0206)$ & $(0.0180)$ \\
\hline Time-varying controls $\left(X_{i s t}\right)$ & $\checkmark$ & $\checkmark$ & $\checkmark$ & $\checkmark$ & $\checkmark$ \\
\hline Time-invariant controls $\left(X_{i s}\right)$ & $\checkmark$ & $\checkmark$ & $\checkmark$ & $\checkmark$ & $\checkmark$ \\
\hline Survey wave controls $(t)$ & $\checkmark$ & $\checkmark$ & $\checkmark$ & $\checkmark$ & $\checkmark$ \\
\hline $\mathrm{N}$ & 216,672 & 204,122 & 204,122 & 203,858 & 203,858 \\
\hline
\end{tabular}

${ }^{*} p<0.05^{* *} p<0.01^{* * *} p<0.001$

Note 1: All models are weighted due to Statistics Canada's RDC disclosure process.

Note 2: Cluster robust standard errors in parentheses. 


\section{MONTEIRO Cultural Assimilation: Learning and Sorting}

\subsection{Further insights}

Source country richness impacts assimilating immigration through exposure, where exposure is determined by sorting into co-ethnic neighbourhoods. The distribution of co-ethnics, or sorting, measure is defined as $\zeta \equiv m^{j} /\left(m^{i}+m^{j}\right)$. The effect of source country richness on the distribution of co-ethnics is given by the estimating equation

$$
\zeta_{i s}=\alpha_{0}+\alpha_{1} h_{i s}+\alpha_{2} X_{i s}+\alpha_{3} X_{i s t}+\alpha_{4} I_{i s}+\epsilon_{i s t}
$$

where $I_{i s}$ is housing costs in CMA/CA of arrival. The regression model (31) is estimated by probit and the results of the estimates are provided in table 6. This result supports the hypothesis of the enclave as a "training system" for new immigrants (Bailey \& Waldinger, 1991; Waldinger, 1993). Those without pre-immigration experience with the local culture are more likely to live and work in the ethnic enclave, although the effects reported are not significant. The ethnic enclave provides new immigrants with an environment that minimizes communication frictions with native-born until the local culture is learned. Furthermore, a recent study by Bredtmann, Nowotny \& Otten (2020) using within-country regional data from the European Union showed that the ability to communicate in the host country language (measured by linguistic distance) and network size are substitutes, such that linguistic distance and size of the linguistic network jointly determine location choices in the host country ${ }^{6}$. This further contributes to the evidence that immigrants coming from more culturally distinct source countries (relative to the host country) rely more on their co-ethnic networks, which is reflected in their location choices in the host country.

Consistent with the results presented in the previous section is a network interpretation of the model. The positive effect of source country richness on assimilating immigration can be explained by greater exposure to co-ethnics. But greater exposure to co-ethnics cannot be solely attributed to size of immigration and clustering of immigrants. There are other factors that determine clustering of co-ethnics; for instance, the qualitative features of the immigrant network must also matter.

Relative RGDP per capita can be interpreted as the immigrant group's average quality. New immigrants that belong to a higher quality group benefit from locating among co-ethnics because the group as a whole assimilates more easily to the local culture. In this sense, quality of the network, rather than size, also plays a role (Edin, Fredriksson \& Aslund, 2003). This is clear from table 6 . Source country richness has a significant positive impact on clustering into co-ethnic communities conditional on size of immigration (i.e. share of co-ethnic group in

${ }^{6}$ I have not accounted for the correlation in size of co-ethnic networks and linguistic distance due to the timing of publication during the COVID-19 lockdown, restricting access to the data at Statistics Canada's Research Data Center. Future research should consider including an interaction between linguistic distance and share of co-ethnics as a control in the model. 
Review of Economic Analysis 13 (2021) 115-156

Table 6: Probit estimation results of living/working in an ethnic enclave: households whose mother tongue is not English and do not reside in Quebec.

\begin{tabular}{lc}
\hline \hline Dependent variable: Living/working in & Pooled \\
an ethnic enclave & $0.211^{* *}$ \\
\hline RGDP per capita $(h)$ & $(0.0771)$ \\
& $0.104^{* * *}$ \\
Housing costs in CMA/CA of arrival $(I)$ & $(0.0309)$ \\
& -0.0920 \\
Informal pre-immigration experience $(\omega)$ & $(0.0736)$ \\
& -0.0892 \\
Formal pre-immigration experience $(\omega)$ & $(0.0782)$ \\
& $6.923^{* * *}$ \\
Share of co-ethnic group in Canada $(m)$ & $(1.648)$ \\
& 0.162 \\
Network $(\beta)$ & $(0.0961)$ \\
& $-0.0268^{* *}$ \\
Population level $(L)$ & $(0.00885)$ \\
& 0.0296 \\
Geographic distance $(\mu)$ & $(0.0207)$ \\
& 0.0862 \\
Linguistic distance $(\theta)$ & $(0.356)$ \\
Months since arrival & -0.0150 \\
\hline Time-varying controls $\left(X_{i s t}\right)$ & $(0.0236)$ \\
\hline N $p<0.05^{* *} p<0.01^{* * *} p<0.001$ & $\checkmark$ \\
$N$ invariant controls $\left(X_{i s}\right)$ & $\checkmark$ \\
\hline wave controls $(t)$ & $\checkmark$ \\
\hline
\end{tabular}

Note 1: All models are weighted due to Statistics Canada's RDC disclosure process.

Note 2: Cluster robust standard errors in parentheses. 


\section{MONTEIRO Cultural Assimilation: Learning and Sorting}

Canada, $m$ ). In addition, other attributes of group quality such as connectedness of the co-ethnic community will also be relevant.

\section{Conclusion}

Immigrants face frictions in communication in their life within the host country due to cultural gaps. Cultural assimilation plays a primary role in determining the success of immigrants by overcoming those cultural barriers. Immigrants assimilate by a process of learning. However, location choices are expected to crowd incentives to further learning so that exposure to other immigrants becomes the primary determinant on cultural accumulation.

Immigrants from poorer source countries have a lower assimilation rate than comparable immigrants from richer countries. This paper has shown that immigrants from poorer source countries have lower assimilation rates because immigrants from poor source countries experience the largest productivity gains from migrating, leading to an increase in their presence within the host country and in turn a greater exposure to co-ethnics.

There are externalities (feedback) associated with the size of immigration within neighbourhoods. Some neighbourhoods will receive more immigrants relative to others. Those neighbourhoods with a large number of immigrants will attract more of the non-assimilating type. Since immigrants from poor source countries are a bigger group, their exposure to co-ethnics will be larger and co-ethnic neighbourhoods will have lower assimilation rates. Among rich source countries total immigration to the host country is smaller. There are fewer externalities and the composition of immigration within neighbourhoods is favorable towards the assimilating type. Immigrants will sort, such that neighbourhoods with fewer immigrants will receive all assimilating types.

Empirically, the implications of the model are tested using longitudinal Canadian data. As predicted, immigrating from a richer source country has a positive effect on assimilating immigration. This effect is observed due to sorting and/or learning. By controlling for learning, the exposure to co-ethnicity channel is not a major determinant of assimilation rates in the host country. However, over-adjusting for the sorting effect, source country richness has a significant positive effect on assimilation rates. There appears to be an alternate channel present. Presumably, this alternate channel is the quality, rather than size, of immigration.

\section{References}

Allen, James P. \& Turner, Eugene (1996). Spatial Pattern of Immigrant Assimilation. Professional Geographer 48(2), 140-155.

Bauer, Thomas; Epstein, Gill S. \& Gang, Ira N. (2005). Enclaves, language, and the location choice of migrants. Journal of Population Economics 18(4), 649-662.

Bailey, Thomas \& Waldinger, Roger (1991). Primary, Secondary, And Enclave Labor Markets: A Training Systems Approach. American Sociological Review 56(4), 432-445. 
Beckhusen, Julia; Florax, Raymond J. G. M. \& de Graaff, Thomas (2012). Living and Working in Ethnic Enclaves: Language Proficiency of Immigrants in U.S. Metropolitan Areas. IZA Discussion Paper Series Discussion Paper No. 6363.

Bonn, Moritz (2012a). Costs and Benefits of Immigration and Multicultural Interaction. MAGKS Joint Discussion Paper Series in Economics 40-2012.

Bonn, Moritz (2012b). Migrants' Acquisition of Cultural Skills and Selective Immigration Policies. MAGKS Joint Discussion Paper Series in Economics 47-2012.

Bredtmann, Julia; Nowotny, Klaus \& Otten, Sebastian (2020). Linguistic distance, networks and migrants' regional location choice. Labour Economics 65, 101863.

Chiswick, Barry R. \& Miller, Paul W. (1996). Ethnic networks and language proficiency among immigrants. Journal of Population Economics 9(1), 19-35.

Chiswick, Barry R. \& Miller, Paul W. (2005). Do Enclaves Matter in Immigrant Adjustment?. City $\mathcal{E}$ Community 4(1), 5-35.

Chiswick, Barry R.; Lee, Yew Liang \& Miller, Paul W. (2004). Immigrants' Language Skills: The Australian Experience in a Longitudinal Survey. International Migration Review 38(2), 611-654.

Damm, Anna Piil (2009). Ethnic Enclaves and Immigrant Labor Market Outcomes: Quasiexperimental Evidence. Journal of Labor Economics 27(2), 281-314.

Danzer, Alexander M.; Feuerbaum, Carsten; Piopiunik, Marc \& Woessmann, Ludger (2018). Growing Up in Ethnic Enclaves: Language Proficiency and Educational Attainment of Immigrant Children. IZA Discussion Paper Series Discussion Paper No. 11608.

Danzer, Alexander M. \& Yaman, Firat (2016). Ethnic concentration and language fluency of immigrants: Evidence from the guest-worker placement in Germany. Journal of Economic Behavior $\mathcal{E}$ Organization 131, 151-165.

Edin, Per-Anders; Frederiksson, Peter \& Aslund, Olof (2003). Ethnic Enclaves and the Economic Success of Immigrants - Evidence from a Natural Experiment. The Quarterly Journal of Economics 118(1), 329-357.

EF EPI (2017). EF English Speaking Proficiency. Education First. Seventh Edition.

Haan, Micheal (2012). The Housing Experiences of New Canadians: Insights from the Longitudinal Survey of Immigrants in Canada (LSIC). Citizenship and Immigration Canada, Research and Evaluation Reference No. RR20120301.

Heston, Alan; Summers, Robert \& Aten, Bettina (2005). Penn World Tables 6.3. Centre for International Comparisons University of Pennsylvania.

Horn, Daniel \& Konya, Istvan (2016). Determinants of the Cultural Integration of Immigrants. In Z. Blaskó (eds.) In Focus: International Migration, 150-158.

Konya, Istvan (2007). Optimal Immigration and Cultural Assimilation. Journal of Labor Economics 25(2), 367-391. 
MONTEIRO Cultural Assimilation: Learning and Sorting

Lazear, Edward P. (1999). Culture and Language. Journal of Political Economy 107(S6), S95S126.

Lazear, Edward P. (2017). Why are Some Immigrant Groups More Successful than Others? NBER Working Paper Series Working Paper No. 23548.

Massey, Douglas S. (1985). Ethnic residential segregation: A theoretical and empirical review. Sociology and Social Research 69(3), 315-350.

Mayer, Thierry \& Zignago, Soledad (2011). Notes on CEPII's distances measure: The GeoDist database. CEPII Working Paper Working Paper No. 2011-25.

Melitz, Jacques \& Toubal, Farid (2014). Native language, spoken language, translation and trade. Journal of International Economics 93(2), 351-363.

Nowotny, Klaus \& Pennerstorfer, Dieter (2019). Network migration: do neighbouring regions matter? Regional Studies 53(1), 107-117.

Pedersen, Peder J.; Pytlikova, Mariola \& Smith, Nina (2008). Selection and network effects Migration flows into OECD 1990-2000. European Economic Review 52, 1160-1186.

Portes, Alejandro \& Shafer, Steven (2006). Revisiting the Enclave Hypothesis: Miami TwentyFive Years Later. The Center for Migration and Development Working Paper Series, Working Paper No. 06-10.

Rabe-Hesketh, Sophia \& Skrondal, Anders (2012). Multilevel and Longitudinal Modeling Using Stata. Volume I: Continuous Responses. STATA Press 3rd Edition.

Rabe-Hesketh, Sophia \& Skrondal, Anders (2012). Multilevel and Longitudinal Modeling Using Stata. Volume II: Categorical Responses, Counts and Survival. STATA Press 3rd Edition.

Statistics Canada (2007). Longitudinal Survey of Immigrants to Canada (LSIC). Statistics Canada. Using York University Research Data Center. Accompanying documentation: Microdata User Guide.

Statistics Canada (2013). Immigration and Ethnocultural Diversity in Canada. Statistics Canada Catalogue no. 99-010-X2011001.

van Tubergen, Frank \& Kalmijn, Matthijs (2009). A Dynamic Approach to the Determinants of Immigrants' Language Proficiency: The United States, 1980-2000. The International Migration Review 43(3), 519-543.

Waldinger, Roger (1993). The Ethnic Enclave Debate Revisited. International Journal of Urban and Regional Research 17(3), 428-436.

Warman, Casey (2007). Ethnic Enclaves and Immigrant Earnings Growth. The Canadian Journal of Economics 40(2), 401-422.

Xie, Yu \& Gough, Margaret (2011). Ethnic Enclaves and the Earnings of Immigrants. Demography 48(4), 1293-1315. 


\section{Appendix}

\subsection{Comparative statics over the thresholds}

The following are a set of useful comparative statics used throughout the main body of the text in derivations, proofs and analysis. The effect of $\theta$ and $m^{i}$ on the threshold for assimilating is given by

$$
\begin{aligned}
& \omega_{a}^{i}=1-\frac{1}{\theta v\left(1+m^{i}\right)}, \quad \frac{\partial \omega_{a}^{i}}{\partial \theta}=\frac{1}{\theta^{2} v\left(1+m^{i}\right)}>0, \quad \frac{\partial \omega_{a}^{i}}{\partial m^{i}}=\frac{1}{\theta v\left(1+m^{i}\right)^{2}}>0 . \\
& \omega_{a}^{j}=1-\frac{1}{\theta v\left(1+m^{j}\right)}, \quad \frac{\partial \omega_{a}^{j}}{\partial \theta}=\frac{1}{\theta^{2} v\left(1+m^{j}\right)}>0, \quad \frac{\partial \omega_{a}^{j}}{\partial m^{j}}=\frac{1}{\theta v\left(1+m^{j}\right)^{2}}>0 .
\end{aligned}
$$

The function $c\left(\beta_{n}\right)$ is the threshold between choosing to locate in neighbourhood $j$ over $i$. The following derivatives provide the effect of changes in $\mu, m^{i}$ and $m^{j}$.

$$
\begin{aligned}
c\left(\beta_{n}\right) & =\frac{m^{j} /\left(1+m^{j}\right)-\left(m^{i} /\left(1+m^{i}\right)\right)\left(\beta_{n} /\left(1-\beta_{n}\right)\right)}{2 \mu}, \\
\frac{\partial c\left(\beta_{n}\right)}{\partial \mu} & =-\frac{m^{j} /\left(1+m^{j}\right)-\left(m^{i} /\left(1+m^{i}\right)\right)\left(\beta_{n} /\left(1-\beta_{n}\right)\right)}{2 \mu^{2}}>0 \text { if } \beta>1 / 2, \\
\frac{\partial c\left(\beta_{n}\right)}{\partial m^{i}} & =-\frac{\beta_{n}}{2 \mu\left(1+m^{i}\right)^{2}\left(1-\beta_{n}\right)}<0, \\
\frac{\partial c\left(\beta_{n}\right)}{\partial m^{j}} & =\frac{1}{2 \mu\left(1+m^{j}\right)^{2}}>0 .
\end{aligned}
$$

The equations $c_{a}^{i}(\omega, \beta)$ and $c_{a}^{j}(\omega, \beta)$ are the thresholds for assimilating immigration in neighbourhoods $i$ and $j$. The following derivatives provide the effect of changes in $h, \mu$ and $\theta$.

$$
\begin{aligned}
c_{a}^{i}(\omega, \beta) & =\frac{1-\theta v(1-\omega)-h / \beta}{\mu}, & c_{a}^{j}(\omega, \beta) & =\frac{1-\theta v(1-\omega)-h /(1-\beta)}{\mu}, \\
\frac{\partial c_{a}^{i}(\omega, \beta)}{\partial h} & =-\frac{1}{\mu \beta}<0, & \frac{\partial c_{a}^{j}(\omega, \beta)}{\partial h} & =-\frac{1}{\mu(1-\beta)}<0, \\
\frac{\partial c_{a}^{i}(\omega, \beta)}{\partial \mu} & =-\frac{1-\theta v(1-\omega)-h / \beta}{\mu^{2}}<0, & \frac{\partial c_{a}^{j}(\omega, \beta)}{\partial \mu} & =-\frac{1-\theta v(1-\omega)-h /(1-\beta)}{\mu^{2}}<0, \\
\frac{\partial c_{a}^{i}(\omega, \beta)}{\partial \theta} & =-\frac{v(1-\omega)}{\mu}<0 . & \frac{\partial c_{a}^{j}(\omega, \beta)}{\partial \theta} & =-\frac{v(1-\omega)}{\mu}<0 .
\end{aligned}
$$

The equations $c_{n}^{i}(\beta)$ and $c_{n}^{j}(\beta)$ are the threshold between non-assimilating immigration in neighbourhoods $i$ and $j$. The following set of derivatives provide the effect of changes in $h, \mu, m^{i}$ 
and/or $m^{j}$.

$$
\begin{aligned}
c_{n}^{i}(\beta) & =\frac{m^{i} /\left(1+m^{i}\right)-h / \beta}{\mu}, & c_{n}^{j}(\beta) & =\frac{m^{j} /\left(1+m^{j}\right)-h /(1-\beta)}{\mu} \\
\frac{\partial c_{n}^{i}(\beta)}{\partial h} & =-\frac{1}{\mu \beta}<0, & \frac{\partial c_{n}^{j}(\beta)}{\partial h} & =-\frac{1}{\mu(1-\beta)}<0, \\
\frac{\partial c_{n}^{i}(\beta)}{\partial \mu} & =-\frac{m^{i} /\left(1+m^{i}\right)-h / \beta}{\mu^{2}}<0, & \frac{\partial c_{n}^{j}(\beta)}{\partial \mu} & =-\frac{m^{j} /\left(1+m^{j}\right)-h /(1-\beta)}{\mu^{2}}, \\
\frac{\partial c_{n}^{i}(\beta)}{\partial m^{i}} & =\frac{1}{\mu\left(1+m^{i}\right)^{2}}>0, & \frac{\partial c_{n}^{j}(\beta)}{\partial m^{j}} & =\frac{1}{\mu\left(1+m^{j}\right)^{2}}>0,
\end{aligned}
$$

\subsection{Comparative statics over $\underline{\omega}^{i}$ and $\underline{\omega}^{j}$}

The equations $\underline{\omega}^{i}$ and $\underline{\omega}^{i}$ are defined as the $\omega$ at which $c_{n}^{i}(\omega)=0$ and $c_{n}^{j}(\omega)=0$. The effect of changes in $h, \theta, m^{i}$ and $m^{j}$ are given as

$$
\begin{aligned}
\underline{\omega}^{i} & =1-\frac{1-h\left(1+m^{i}\left(1+m^{j}\right) / m^{j}\left(1+m^{i}\right)\right)}{\theta v}, & \underline{\omega}^{j} & =1-\frac{1-h\left(1+m^{j}\left(1+m^{i}\right) / m^{i}\left(1+m^{j}\right)\right)}{\theta v}, \\
\frac{\partial \underline{\omega}^{i}}{\partial h} & =\frac{1+m^{i}\left(1+m^{j}\right) / m^{j}\left(1+m^{i}\right)}{\theta v}>0, & \frac{\partial \underline{\omega}^{j}}{\partial h} & =\frac{1+m^{j}\left(1+m^{i}\right) / m^{i}\left(1+m^{j}\right)}{\theta v}>0, \\
\frac{\partial \underline{\omega}^{i}}{\partial m^{i}} & =\frac{h}{\theta v} \frac{1+m^{j}}{m^{j}\left(1+m^{i}\right)^{2}}>0, & \frac{\partial \underline{\omega}^{j}}{\partial m^{i}} & =-\frac{h}{\theta v} \frac{m^{j}}{\left(m^{i}\right)^{2}\left(1+m^{j}\right)}<0, \\
\frac{\partial \underline{\omega}^{i}}{\partial m^{j}} & =-\frac{h}{\theta v} \frac{m^{i}}{\left(m^{j}\right)^{2}\left(1+m^{i}\right)}<0, & \frac{\partial \underline{\omega}^{j}}{\partial m^{i}} & =\frac{h}{\theta v} \frac{1+m^{i}}{m^{i}\left(1+m^{j}\right)^{2}}>0, \\
\frac{\partial \underline{\omega}^{i}}{\partial \theta} & =\frac{1-h\left(1+m^{i}\left(1+m^{j}\right) / m^{j}\left(1+m^{i}\right)\right.}{\theta^{2} v}<0, & \frac{\partial \underline{\omega}^{j}}{\partial \theta} & =\frac{1-h\left(1+m^{j}\left(1+m^{i}\right) / m^{i}\left(1+m^{j}\right)\right.}{\theta^{2} v}<0,
\end{aligned}
$$

\subsection{Comparative statics over $\Gamma^{i}\left(m^{i}, m^{j}\right)$ and $\Gamma^{j}\left(m^{i}, m^{j}\right)$}

Consider a mixed equilibrium outcome $k(1-\omega) \leq 1, \omega_{a}^{i} \geq \underline{\omega}^{i}$ and $\omega_{a}^{j}>\underline{\omega}^{j}$. The elements of the Jacobian matrix $J\left(m^{i}, m^{j}\right)$ are the set of first-order partial differentials of $\Gamma^{i}\left(m^{i}, m^{j}\right)$ and 
Review of Economic Analysis 13 (2021) 115-156

$\Gamma^{j}\left(m^{i}, m^{j}\right)$ in terms of $m^{i}$ and $m^{j}$. As follows,

$$
\begin{aligned}
& m^{i}=L \int_{\omega_{a}^{i}}^{1} \int_{1 / 2}^{1} F\left(c_{a}^{i}(\omega, \beta)\right) d B(\beta) d W(\omega) \\
& +L W\left(\omega_{a}^{i}\right)^{2} \int_{\frac{h\left(1+m^{i}\right)}{m^{i}}}^{1} \max \left\{0, F\left(c_{n}^{i}(\beta)\right)-F\left(c\left(\beta_{n}\right)\right)\right\} d B(\beta) \equiv \Gamma^{i}\left(m^{i}, m^{j}\right) \\
& \frac{\partial \Gamma^{i}\left(m^{i}, m^{j}\right)}{\partial m^{i}}=-L \int_{1 / 2}^{1} F\left(c_{a}^{i}(\omega, \beta)\right) d B(\beta) \frac{\partial \omega_{a}^{i}}{\partial m^{i}} \\
& +2 L W\left(\omega_{a}^{i}\right) W^{\prime}\left(\omega_{a}^{i}\right) \frac{\partial \omega_{a}^{i}}{\partial m^{i}} \int_{\frac{h\left(1+m^{i}\right)}{m^{i}}}^{1} \max \left\{0, F\left(c_{n}^{i}(\beta)\right)-F\left(c\left(\beta_{n}\right)\right)\right\} d B(\beta) \\
& +L W\left(\omega_{a}^{i}\right)^{2} \int_{\frac{h\left(1+m^{i}\right)}{m^{i}}}^{1} \max \left\{0, f\left(c_{n}^{i}(\beta)\right) \frac{\partial c_{n}^{i}(\beta)}{\partial m^{i}}-f\left(c\left(\beta_{n}\right)\right) \frac{\partial c\left(\beta_{n}\right)}{\partial m^{i}}\right\} d B(\beta) \\
& \frac{\partial \Gamma^{i}\left(m^{i}, m^{j}\right)}{\partial m^{i}}=L W\left(\omega_{a}^{i}\right)^{2} \int_{\frac{h\left(1+m^{i}\right)}{m^{i}}}^{1} \max \left\{0,-f\left(c\left(\beta_{n}\right)\right) \frac{\partial c\left(\beta_{n}\right)}{\partial m^{j}}\right\} d B(\beta)=0 \\
& m^{j}=L \int_{\omega_{a}^{j}}^{1} \int_{0}^{1 / 2} F\left(c_{a}^{j}(\omega, \beta)\right) d B(\beta) d W(\omega) \\
& +L\left(W\left(\omega_{a}^{j}\right)-W\left(\underline{\omega}^{j}\right)\right)^{2} \int_{0}^{\frac{m^{j}\left(1+m^{i}\right)}{m^{i}+m^{j}+2 m^{i} m^{j}}} F\left(c\left(\beta_{n}\right)\right) d B(\beta) \equiv \Gamma^{j}\left(m^{i}, m^{j}\right) \\
& \frac{\partial \Gamma^{j}\left(m^{i}, m^{j}\right)}{\partial m^{i}}=-2 L\left(W\left(\omega_{a}^{j}\right)-W\left(\underline{\omega}^{j}\right)\right) W^{\prime}\left(\underline{\omega}^{j}\right) \frac{\partial \underline{\omega}^{j}}{\partial m^{i}} \int_{0}^{\frac{m^{j}\left(1+m^{i}\right)}{m^{i}+m^{j}+2 m^{i} m^{j}}} F\left(c\left(\beta_{n}\right)\right) d B(\beta) \\
& +L\left(W\left(\omega_{a}^{j}\right)-W\left(\underline{\omega}^{j}\right)\right)^{2} \int_{0}^{\frac{m^{j}\left(1+m^{i}\right)}{m^{i}+m^{j}+2 m^{i} m^{j}}} f\left(c\left(\beta_{n}\right)\right) \frac{\partial c\left(\beta_{n}\right)}{\partial m^{i}} d B(\beta) \\
& \frac{\partial \Gamma^{j}\left(m^{i}, m^{j}\right)}{\partial m^{j}}=-L \int_{0}^{1 / 2} F\left(c_{a}^{j}(\omega, \beta)\right) d B(\beta) \frac{\partial \omega_{a}^{j}}{\partial m^{j}} \\
& +2 L\left(W\left(\omega_{a}^{j}\right)-W\left(\underline{\omega}^{j}\right)\right)\left(W^{\prime}\left(\omega_{a}^{j}\right) \frac{\partial \omega_{a}^{j}}{\partial m^{j}}-W^{\prime}\left(\underline{\omega}^{j}\right) \frac{\partial \underline{\omega}^{j}}{\partial m^{j}}\right) \int_{0}^{\frac{m^{j}\left(1+m^{i}\right)}{m^{i}+m^{j}+2 m^{i} m^{j}}} F\left(c\left(\beta_{n}\right)\right) d B(\beta) \\
& +L\left(W\left(\omega_{a}^{j}\right)-W\left(\underline{\omega}^{j}\right)\right)^{2} \int_{0}^{\frac{m^{j}\left(1+m^{i}\right)}{m^{i}+m^{j}+2 m^{i} m^{j}}} f\left(c\left(\beta_{n}\right)\right) \frac{\partial c\left(\beta_{n}\right)}{\partial m^{j}} d B(\beta)
\end{aligned}
$$

Since $\Gamma^{i}\left(m^{i}, m^{j}\right)$ and $\Gamma^{j}\left(m^{i}, m^{j}\right)$ are also directly affected by the parameters $\{L, h, \mu, \theta\}$. These 


\section{MONTEIRO Cultural Assimilation: Learning and Sorting}

comparative statics are provided as well in the mixed equilibrium case.

$$
\begin{aligned}
& \frac{\partial \Gamma^{i}\left(m^{i}, m^{j}\right)}{\partial L}=\int_{\omega_{a}^{i}}^{1} \int_{1 / 2}^{1} F\left(c_{a}^{i}(\omega, \beta)\right) d B(\beta) d W(\omega) \\
& +W\left(\omega_{a}^{i}\right)^{2} \int_{\frac{h\left(1+m^{i}\right)}{m^{i}}}^{1} \max \left\{0, F\left(c_{n}^{i}(\beta)\right)-F\left(c\left(\beta_{n}\right)\right)\right\} d B(\beta)>0 \\
& \frac{\partial \Gamma^{i}\left(m^{i}, m^{j}\right)}{\partial h}=L \int_{\omega_{a}^{i}}^{1} \int_{1 / 2}^{1} f\left(c_{a}^{i}(\omega, \beta)\right) \frac{\partial c_{a}^{i}(\omega, \beta)}{\partial h} d B(\beta) d W(\omega)<0 \\
& \frac{\partial \Gamma^{i}\left(m^{i}, m^{j}\right)}{\partial \mu}=L \int_{\omega_{a}^{i}}^{1} \int_{1 / 2}^{1} f\left(c_{a}^{i}(\omega, \beta)\right) \frac{\partial c_{a}^{i}(\omega, \beta)}{\partial \mu} d B(\beta) d W(\omega)<0 \\
& \frac{\partial \Gamma^{i}\left(m^{i}, m^{j}\right)}{\partial \theta}=-L \int_{1 / 2}^{1} F\left(c_{a}^{i}(\omega, \beta)\right) d B(\beta) \frac{\partial \omega_{a}^{i}}{\partial \theta}+L \int_{\omega_{a}^{i}}^{1} \int_{1 / 2}^{1} f\left(c_{a}^{i}(\omega, \beta)\right) \frac{\partial c_{a}^{i}(\omega, \beta)}{\partial \theta} d B(\beta) d W(\omega) \\
& +2 L W\left(\omega_{a}^{i}\right)^{2} W^{\prime}\left(\omega_{a}^{i}\right) \frac{\partial \omega_{a}^{i}}{\partial \theta} \int_{\frac{h\left(1+m^{i}\right)}{m^{i}}}^{1} \max \left\{0, F\left(c_{n}^{i}(\beta)\right)-F\left(c\left(\beta_{n}\right)\right)\right\} d B(\beta) \lesseqgtr 0 \\
& \frac{\partial \Gamma^{j}\left(m^{i}, m^{j}\right)}{\partial L}=\int_{\omega_{a}^{j}}^{1} \int_{0}^{1 / 2} F\left(c_{a}^{j}(\omega, \beta)\right) d B(\beta) d W(\omega) \\
& +\left(W\left(\omega_{a}^{j}\right)-W\left(\underline{\omega}^{j}\right)\right)^{2} \int_{0}^{\frac{m^{j}\left(1+m^{i}\right)}{m^{i}+m^{j}+2 m^{i} m^{j}}} F\left(c\left(\beta_{n}\right)\right) d B(\beta)>0 \\
& \frac{\partial \Gamma^{j}\left(m^{i}, m^{j}\right)}{\partial h}=L \int_{\omega_{a}^{j}}^{1} \int_{0}^{1 / 2} f\left(c_{a}^{j}(\omega, \beta)\right) \frac{\partial c_{a}^{j}(\omega, \beta)}{\partial h} d B(\beta) d W(\omega) \\
& -2 L\left(W\left(\omega_{a}^{j}\right)-W\left(\underline{\omega}^{j}\right)\right) W^{\prime}\left(\underline{\omega}^{j}\right) \frac{\partial \underline{\omega}^{j}}{\partial h} \int_{0}^{\frac{m^{j}\left(1+m^{i}\right)}{m^{i}+m^{j}+2 m^{i} m}} F\left(c\left(\beta_{n}\right)\right) d B(\beta)<0 \\
& \frac{\partial \Gamma^{j}\left(m^{i}, m^{j}\right)}{\partial \mu}=L \int_{\omega_{a}^{j}}^{1} \int_{0}^{1 / 2} f\left(c_{a}^{j}(\omega, \beta)\right) \frac{\partial c_{a}^{j}(\omega, \beta)}{\partial \mu} d B(\beta) d W(\omega) \\
& +L\left(W\left(\omega_{a}^{j}\right)-W\left(\underline{\omega}^{j}\right)\right)^{2} \int_{0}^{\frac{m^{j}\left(1+m^{i}\right)}{m^{i}+m^{j}+2 m^{i} m^{j}}} f\left(c\left(\beta_{n}\right)\right) \frac{\partial c\left(\beta_{n}\right)}{\partial \mu} d B(\beta)<0 \\
& \frac{\partial \Gamma^{j}\left(m^{i}, m^{j}\right)}{\partial \theta}=-L \int_{0}^{1 / 2} F\left(c_{a}^{j}(\omega, \beta)\right) d B(\beta) \frac{\partial \omega_{a}^{j}}{\partial \theta}+L \int_{\omega_{a}^{j}}^{1} \int_{0}^{1 / 2} f\left(c_{a}^{j}(\omega, \beta)\right) \frac{\partial c_{a}^{j}(\omega, \beta)}{\partial \theta} d B(\beta) d W(\omega) \\
& +2 L\left(W\left(\omega_{a}^{j}\right)-W\left(\underline{\omega}^{j}\right)\right)\left(W^{\prime}\left(\omega_{a}^{j}\right) \frac{\partial \omega_{a}^{j}}{\partial \theta}-W^{\prime}\left(\underline{\omega}^{j}\right) \frac{\partial \underline{\omega}^{j}}{\partial \theta}\right) \int_{0}^{\frac{m^{j}\left(1+m^{i}\right)}{m^{i}+m^{j}+2 m^{i} m^{j}}} F\left(c\left(\beta_{n}\right)\right) d B(\beta) \lesseqgtr 0
\end{aligned}
$$

In a sorting equilibrium outcome $k(1-\omega) \leq 1, \omega_{a}^{i} \geq \underline{\omega}^{i}$ and $\omega_{a}^{j}=\underline{\omega}^{j}$. The first-order differentials of $\Gamma^{i}\left(m^{i}, m^{j}\right)$ in terms of $m^{i}$ and $m^{j}$, and $\{L, h, \mu, \theta\}$ are the same as in the mixed 
case. But the set of first-order differentials of $\Gamma^{j}\left(m^{i}, m^{j}\right)$ are as follows,

$$
\begin{aligned}
& \frac{\partial \Gamma^{j}\left(m^{i}, m^{j}\right)}{\partial m^{i}}=-L \int_{0}^{1 / 2} F\left(c_{a}^{j}(\omega, \beta)\right) d B(\beta) \frac{\partial \underline{\omega}^{j}}{\partial m^{i}}<0 \\
& \frac{\partial \Gamma^{j}\left(m^{i}, m^{j}\right)}{\partial m^{j}}=-L \int_{0}^{1 / 2} F\left(c_{a}^{j}(\omega, \beta)\right) d B(\beta) \frac{\partial \underline{\omega}^{j}}{\partial m^{j}}<0 \\
& \frac{\partial \Gamma^{j}\left(m^{i}, m^{j}\right)}{\partial L}=\int_{\underline{\omega}^{j}}^{1} \int_{0}^{1 / 2} F\left(c_{a}^{j}(\omega, \beta)\right) d B(\beta) d W(\omega)>0 \\
& \frac{\partial \Gamma^{j}\left(m^{i}, m^{j}\right)}{\partial h}=-L \int_{0}^{1 / 2} F\left(c_{a}^{j}(\omega, \beta)\right) d B(\beta) \frac{\partial \underline{\omega}^{j}}{\partial h}+L \int_{\underline{\omega}^{j}}^{1} \int_{0}^{1 / 2} f\left(c_{a}^{j}(\omega, \beta)\right) \frac{\partial c_{a}^{j}(\omega, \beta)}{\partial h} d B(\beta) d W(\omega)<0 \\
& \frac{\partial \Gamma^{j}\left(m^{i}, m^{j}\right)}{\partial \mu}=L \int_{\underline{\omega}^{j}}^{1} \int_{0}^{1 / 2} f\left(c_{a}^{j}(\omega, \beta)\right) \frac{\partial c_{a}^{j}(\omega, \beta)}{\partial \mu} d B(\beta) d W(\omega)<0 \\
& \frac{\partial \Gamma^{j}\left(m^{i}, m^{j}\right)}{\partial \theta}=-L \int_{0}^{1 / 2} F\left(c_{a}^{j}(\omega, \beta)\right) d B(\beta) \frac{\partial \underline{\omega}^{j}}{\partial \theta}+L \int_{\underline{\omega}^{j}}^{1} \int_{0}^{1 / 2} f\left(c_{a}^{j}(\omega, \beta)\right) \frac{\partial c_{a}^{j}(\omega, \beta)}{\partial \theta} d B(\beta) d W(\omega) \lesseqgtr 0
\end{aligned}
$$

\subsection{Comparative statics over $m^{i}(L, h, \mu, \theta)$ and $m^{j}(L, h, \mu, \theta)$}

Since $m^{i}=\Gamma^{i}\left(m^{i}, m^{j} ; \mathbb{P}\right)$ and $m^{j}=\Gamma^{j}\left(m^{i}, m^{j} ; \mathbb{P}\right)$ implicitly define $m^{i}$ and $m^{j}$, where $\mathbb{P} \equiv$ $\{L, h, \mu, \theta\}$. Then totally differentiating with respect to the parameters in $\mathbb{P}$ is given by

$$
\begin{aligned}
\frac{d m^{i}}{d \mathbb{P}} & =\frac{\partial \Gamma^{i}}{\partial m^{i}} \frac{\partial m^{i}}{\partial \mathbb{P}}+\frac{\partial \Gamma^{i}}{\partial m^{j}} \frac{\partial m^{j}}{\partial \mathbb{P}}+\frac{\partial \Gamma^{i}}{\partial \mathbb{P}} \\
\frac{d m^{j}}{d \mathbb{P}} & =\frac{\partial \Gamma^{j}}{\partial m^{i}} \frac{\partial m^{i}}{\partial \mathbb{P}}+\frac{\partial \Gamma^{j}}{\partial m^{j}} \frac{\partial m^{j}}{\partial \mathbb{P}}+\frac{\partial \Gamma^{j}}{\partial \mathbb{P}}
\end{aligned}
$$

Solving the two equation systems simultaneously provides the following set of reduced form solutions to the differentials of $m^{i}$ and $m^{j}$ in terms of $\mathbb{P}$.

$$
\begin{aligned}
\frac{d m^{i}}{d \mathbb{P}} & =\frac{\partial \Gamma^{i} / \partial m^{j} \times \partial \Gamma^{j} / \partial \mathbb{P}+\partial \Gamma^{i} / \partial \mathbb{P} \times\left(1-\partial \Gamma^{j} / \partial m^{j}\right)}{\left(1-\partial \Gamma^{i} / \partial m^{i}\right)\left(1-\partial \Gamma^{j} / \partial m^{j}\right)-\partial \Gamma^{i} / \partial m^{j} \times \partial \Gamma^{j} / \partial m^{i}} \\
\frac{d m^{j}}{d \mathbb{P}} & =\frac{\partial \Gamma^{j} / \partial m^{i} \times d m^{i} / d \mathbb{P}+\partial \Gamma^{j} / \partial \mathbb{P}}{1-\partial \Gamma^{j} / \partial m^{j}}
\end{aligned}
$$

The signs of the partial differentials, $\partial \Gamma^{i} / \partial \mathbb{P}$ and $\partial \Gamma^{j} / \partial \mathbb{P}$, have already been determined in the previous section. Additionally, $\partial \Gamma^{i} / \partial m^{j}=0$ which simplifies the problem further. Moreover, the stability conditions require that $\partial \Gamma^{i} / \partial m^{i}<0$ and $\partial \Gamma^{j} / \partial m^{j}<0$. Finally, $\partial \Gamma^{j} / \partial m^{i}>0$ is also implied by the stability conditions. Using this information the effect of $\mathbb{P}$ on $m^{i}$ and $m^{j}$ in the mixed and sorting cases are given as

$$
\begin{aligned}
& \frac{d m^{i}}{d L}>0, \quad \frac{d m^{i}}{d h}<0, \quad \frac{d m^{i}}{d \mu}<0, \quad \frac{d m^{i}}{d \theta} \lesseqgtr 0, \\
& \frac{d m^{j}}{d L}>0, \quad \frac{d m^{j}}{d h}<0, \quad \frac{d m^{j}}{d \mu}<0, \quad \frac{d m^{j}}{d \theta} \lesseqgtr 0 .
\end{aligned}
$$




\section{MONTEIRO Cultural Assimilation: Learning and Sorting}

\subsection{Direct effects}

The direct effects of table 2 are determined by changes in $\mathbb{P}=\{L, h, \mu, \theta\}$ on $\rho^{j}=a^{j} / m^{j}$. To simplify the calculations, let $\eta \equiv \mathbb{A} / \mathbb{B}$, where

$$
\begin{aligned}
& \mathbb{A}=L \int_{\omega_{a}^{j}}^{1} \int_{0}^{1 / 2} F\left(c_{a}^{j}(\omega, \beta)\right) d B(\beta) d W(\omega)>0 \\
& \mathbb{B}=L\left(W\left(\omega_{a}^{j}\right)-W\left(\underline{\omega}^{j}\right)\right) \int_{0}^{\frac{m^{j}\left(1+m^{i}\right)}{m^{i}+m^{j}+2 m^{i} m^{j}}} F\left(c\left(\beta_{n}\right)\right) d B(\beta)>0
\end{aligned}
$$

Now $\rho^{j}$ can be written in a simpler form, as a function of $\eta$. It can then be inferred that $\rho^{j}$ is a monotonic increasing function of $\eta$.

$$
\rho^{j}=\frac{\eta+\int_{\underline{\omega}^{j}}^{\omega^{j}} k(1-\omega) d W(\omega)}{\eta+\left(W\left(\omega_{a}^{j}\right)-W\left(\underline{\omega}^{j}\right)\right)}, \quad \frac{\partial \rho^{j}}{\partial \eta}>0 \quad \forall k(1-\omega) \leq 1
$$

The parameters in the model $\mathbb{P}=\{L, h, \mu, \theta\}$ are expected to have a direct effect on $\rho^{j}=a^{j} / \mathrm{m}^{j}$. These effects are estimated by the relationship

$$
\frac{\partial \rho^{j}}{\partial \mathbb{P}}=\frac{\partial \rho^{j}}{\partial \eta} \times \frac{\partial \eta}{\partial \mathbb{P}}, \quad \text { where } \quad \frac{\partial \eta}{\partial \mathbb{P}}=\frac{\partial \mathbb{A} / \partial \mathbb{P} \times \mathbb{B}-\partial \mathbb{B} / \partial \mathbb{P} \times \mathbb{A}}{\mathbb{B}^{2}} .
$$

All that remains to sign the direct effect is differentiating $\mathbb{A}$ and $\mathbb{B}$ in terms of the elements in $\mathbb{P}=\{L, h, \mu, \theta\}$.

$$
\begin{aligned}
\frac{\partial \mathbb{A}}{\partial L} & =\int_{\omega_{a}^{j}}^{1} \int_{0}^{1 / 2} F\left(c_{a}^{j}(\omega, \beta)\right) d B(\beta) d W(\omega)>0, \\
\frac{\partial \mathbb{A}}{\partial h} & =L \int_{\omega_{a}^{j}}^{1} \int_{0}^{1 / 2} f\left(c_{a}^{j}(\omega, \beta)\right) \frac{\partial c_{a}^{j}(\omega, \beta)}{\partial h} d B(\beta) d W(\omega)<0, \\
\frac{\partial \mathbb{A}}{\partial \mu} & =L \int_{\omega_{a}^{j}}^{1} \int_{0}^{1 / 2} f\left(c_{a}^{j}(\omega, \beta)\right) \frac{\partial c_{a}^{j}(\omega, \beta)}{\partial \mu} d B(\beta) d W(\omega)<0, \\
\frac{\partial \mathbb{A}}{\partial \theta} & =-L \int_{0}^{1 / 2} F\left(c_{a}^{j}(\omega, \beta)\right) d B(\beta) \frac{\partial \omega_{a}^{j}}{\partial \theta} d W(\omega)+L \int_{\omega_{a}^{j}}^{1} \int_{0}^{1 / 2} f\left(c_{a}^{j}(\omega, \beta)\right) \frac{\partial c_{a}^{j}(\omega, \beta)}{\partial \theta} d B(\beta) d W(\omega)<0, \\
\frac{\partial \mathbb{A}}{\partial m^{i}} & =0, \\
\frac{\partial \mathbb{A}}{\partial m^{j}} & =-L \int_{0}^{1 / 2} F\left(c_{a}^{j}(\omega, \beta)\right) \frac{\partial \omega_{a}^{j}}{\partial m^{j}} d B(\beta) d W(\omega)<0,
\end{aligned}
$$


Review of Economic Analysis 13 (2021) 115-156

$$
\begin{aligned}
\frac{\partial \mathbb{B}}{\partial L}= & \left(W\left(\omega_{a}^{j}\right)-W\left(\underline{\omega}^{j}\right)\right) \int_{0}^{\frac{m^{j}\left(1+m^{i}\right)}{m^{i}+m^{j}+2 m^{i} m^{j}}} F\left(c\left(\beta_{n}\right)\right) d B(\beta)>0, \\
\frac{\partial \mathbb{B}}{\partial h}= & -L W^{\prime}\left(\underline{\omega}^{j}\right) \frac{\partial \underline{\omega}^{j}}{\partial h} \int_{0}^{\frac{m^{j}\left(1+m^{i}\right)}{m^{i}+m^{j}+2 m^{i} m^{j}}} F\left(c\left(\beta_{n}\right)\right) d B(\beta)<0, \\
\frac{\partial \mathbb{B}}{\partial \mu}= & L\left(W\left(\omega_{a}^{j}\right)-W\left(\underline{\omega}^{j}\right)\right) \int_{0}^{\frac{m^{j}\left(1+m^{i}\right)}{m^{i}+m^{j}+2 m^{i} m^{j}}} f\left(c\left(\beta_{n}\right)\right) \frac{\partial c\left(\beta_{n}\right)}{\partial \mu} d B(\beta)>0, \\
\frac{\partial \mathbb{B}}{\partial \theta}= & L\left(W^{\prime}\left(\omega_{a}^{j}\right) \frac{\partial \omega_{a}^{j}}{\partial \theta}-W^{\prime}\left(\underline{\omega}^{j}\right) \frac{\partial \underline{\omega}^{j}}{\partial \theta}\right) \int_{0}^{\frac{m^{j}\left(1+m^{i}\right)}{m^{i}+m^{j}+2 m^{i} m^{j}}} F\left(c\left(\beta_{n}\right)\right) d B(\beta) \lesseqgtr 0, \\
\frac{\partial \mathbb{B}}{\partial m^{i}}= & -L W^{\prime}\left(\underline{\omega}^{j}\right) \frac{\partial \underline{\omega}^{j}}{\partial m^{i}} \int_{0}^{\frac{m^{j}\left(1+m^{i}\right)}{m^{i}+m^{j}+2 m^{i} m^{j}}} F\left(c\left(\beta_{n}\right)\right) d B(\beta) \\
& +L\left(W\left(\omega_{a}^{j}\right)-W\left(\underline{\omega}^{j}\right)\right) \int_{0}^{\frac{m^{j}\left(1+m^{i}\right)}{m^{i}+m^{j}+2 m^{i} m^{j}}} f\left(c\left(\beta_{n}\right)\right) \frac{\partial c\left(\beta_{n}\right)}{\partial m^{i}} d B(\beta) \lesseqgtr 0, \\
\frac{\partial \mathbb{B}}{\partial m^{j}}= & L\left(W^{\prime}\left(\omega_{a}^{j}\right) \frac{\partial \omega_{a}^{j}}{\partial m^{j}}-W^{\prime}\left(\underline{\omega}^{j}\right) \frac{\partial \underline{\omega}^{j}}{\partial m^{j}}\right) \int_{0}^{\frac{m^{j}\left(1+m^{i}\right)}{m^{i}+m^{j}+2 m^{i} m^{j}}} F\left(c\left(\beta_{n}\right)\right) d B(\beta) \\
& \quad+L\left(W\left(\omega_{a}^{j}\right)-W\left(\underline{\omega}^{j}\right)\right) \int_{0}^{\frac{m^{j}\left(1+m^{i}\right)}{m^{i}+m^{j}+2 m^{i} m^{j}}} f\left(c\left(\beta_{n}\right)\right) \frac{\partial c\left(\beta_{n}\right)}{\partial m^{j}} d B(\beta) \lesseqgtr 0 .
\end{aligned}
$$

\subsection{Indirect effects}

The indirect effects of table 2 are determined by changes in $\mathbb{P}=\{L, h, \mu, \theta\}$ on $\rho^{j}=a^{j} / m^{j}$ through changes in $m^{i}(L, h, \mu, \theta)$. These effects are estimated by the relationship

$$
\frac{\partial \rho^{j}}{\partial \mathbb{P}}=\frac{\partial \rho^{j}}{\partial \eta} \times \frac{\partial \eta}{\partial m^{i}} \times \frac{\partial m^{i}}{\partial \mathbb{P}}, \quad \text { where } \quad \frac{\partial \eta}{\partial m^{i}}=\frac{\partial \mathbb{A} / \partial m^{i} \times \mathbb{B}-\partial \mathbb{B} / \partial m^{i} \times \mathbb{A}}{\mathbb{B}^{2}} .
$$

But since $\partial \mathbb{B} / \partial m^{i}$ was ambiguously signed, all the indirect effects through $m^{i}(L, h, \mu, \theta)$ are ambiguous as well. Similarly, I can estimate the indirect effects through $m^{j}(L, h, \mu, \theta)$ as

$$
\frac{\partial \rho^{j}}{\partial \mathbb{P}}=\frac{\partial \rho^{j}}{\partial \eta} \times \frac{\partial \eta}{\partial m^{j}} \times \frac{\partial m^{j}}{\partial \mathbb{P}}, \quad \text { where } \quad \frac{\partial \eta}{\partial m^{j}}=\frac{\partial \mathbb{A} / \partial m^{j} \times \mathbb{B}-\partial \mathbb{B} / \partial m^{j} \times \mathbb{A}}{\mathbb{B}^{2}} .
$$

Again, $\partial \mathbb{B} / \partial m^{j}$ is ambiguously signed, all the indirect effects through $m^{j}(L, h, \mu, \theta)$ are ambiguous. The results are depicted as such in table 2 .

\subsection{Threshold effects}

The threshold effects are identified by changes in $\mathbb{P}=\{L, h, \mu, \theta\}$ on equation (25), or $\phi($.$) . These$ effects are provided below.

$$
\frac{d \phi}{d L}<0, \quad \frac{d \phi}{d h}>0, \quad \frac{d \phi}{d \mu}>0, \quad \frac{d \phi}{d \theta} \lesseqgtr 0 .
$$

\subsection{Tables}


Table 8: Description of variables used in this study.

\begin{tabular}{|c|c|}
\hline Variable Name & Description \\
\hline Speaking proficiency & English speaking proficiency of the respondent (5-level categorical) \\
\hline Ethnic enclave & $\begin{array}{l}\text { Arrived in a CMA/CA that is overrepresented by co-ethnics } \\
\text { (Census of Population 2001), and worked in an organization that is } \\
\text { mostly co-ethnic (Dummy) }\end{array}$ \\
\hline $\begin{array}{l}\text { Housing costs in CMA/CA } \\
\text { of arrival (in \$'000's) }\end{array}$ & $\begin{array}{l}\text { Average cost of housing in CMA/CA of arrival. (Continuous; } \\
\text { Census of Population 2001) }\end{array}$ \\
\hline Formal investment & Learned to speak English in language class or school (Dummy) \\
\hline Informal investment & $\begin{array}{l}\text { Learned to speak English through family \& friends, work, } \\
\text { non-language classes, media, self-study and/or other (Dummy) }\end{array}$ \\
\hline CMA of arrival: Toronto & $\begin{array}{l}\text { The respondent lives in Toronto at the time of landing in Canada } \\
\text { (Dummy) }\end{array}$ \\
\hline $\begin{array}{l}\text { CMA of arrival: } \\
\text { Vancouver }\end{array}$ & $\begin{array}{l}\text { The respondent lived in Vancouver at the time of landing in Canada } \\
\text { (Dummy) }\end{array}$ \\
\hline $\begin{array}{l}\text { Share of co-ethnic group } \\
\text { in Canada }\end{array}$ & $\begin{array}{l}\text { Share of the co-ethnic population that the respondent belongs to } \\
\text { admitted into Canada in } 2000 \text { (Continuous) }\end{array}$ \\
\hline Education outside Canada: & Highest level of formal education attained outside of Canada is \\
\hline Bachelor's or higher & Bachelor's or higher (Dummy) \\
\hline $\begin{array}{l}\text { Formal learning of English } \\
\text { outside of Canada }\end{array}$ & $\begin{array}{l}\text { Learned most of their English before coming to Canada through } \\
\text { language classes, a private tutor or schooling (Dummy) }\end{array}$ \\
\hline Informal learning of & Learned most of their English before coming to Canada from \\
\hline English outside of Canada & $\begin{array}{l}\text { family/friends, self-study, work, media, everyday interactions or } \\
\text { other (Dummy) }\end{array}$ \\
\hline Friend/family networks & $\begin{array}{l}\text { Respondent had friends/family in current area (or nearby area) and } \\
\text { chose this area because friends/family live here, in wave } 1 \\
\text { (Dummy) }\end{array}$ \\
\hline Age at immigration & Age of the respondent (Continuous) \\
\hline Male & Gender of the respondent $=$ Male $($ Dummy $)$ \\
\hline Married & Marital status of the respondent $=$ Married (Dummy) \\
\hline Employed & The respondent is employed full-time (Dummy) \\
\hline $\begin{array}{l}\text { Savings brought from } \\
\text { outside Canada }\end{array}$ & Total amount of savings brough from outside Canada (Continuous) \\
\hline Family class & Immigration category $=$ Family class (Dummy) \\
\hline $\begin{array}{l}\text { Number of immigrating } \\
\text { members }\end{array}$ & Size of the immigrating party at the time of landing (Continuous) \\
\hline
\end{tabular}


Review of Economic Analysis 13 (2021) 115-156

Table 8: Description of variables used in this study.

\begin{tabular}{ll}
\hline Variable Name & Description \\
\hline $\begin{array}{l}\text { Number of joining } \\
\text { members } \\
\text { member of household }\end{array}$ & $\begin{array}{l}\text { Number of members that have joined the household since landing } \\
\text { (wave 1) or since the last interview (waves 2 and 3) (Continuous) } \\
\text { Size of household (Continuous) }\end{array}$ \\
$\begin{array}{l}\text { Monther of children } \\
\text { RGDP in place of Birth }\end{array}$ & $\begin{array}{l}\text { Percentage of children living in the household (Continuous) } \\
\text { Number of months elapsed since landing in Canada (Continuous) } \\
\text { Real GDP of place of birth of the respondent relative to Canada } \\
\text { (Continuous; Heston, Summers \& Aten, 2005) }\end{array}$ \\
Population in place of & $\begin{array}{l}\text { Population of place of birth of the respondent relative to Canada } \\
\text { (Continuous; Heston, Summers \& Aten, 2005) } \\
\text { birth }\end{array}$ \\
$\begin{array}{l}\text { Distance from capital city } \\
\text { in place of birth (in '000's) of the capital city in the place of birth of the respondent } \\
\text { Linguistic distance }\end{array}$ & $\begin{array}{l}\text { Linguistic distance of the official language in the place of birth of } \\
\text { the respondent relative to English (Continuous; Melitz \& Toubal, } \\
\text { 2014) }\end{array}$ \\
\hline
\end{tabular}

Table 9: Summary statistics.

\begin{tabular}{|c|c|c|c|c|c|c|}
\hline \multirow[b]{2}{*}{ Variable Name } & \multicolumn{3}{|c|}{ Full sample } & \multicolumn{3}{|c|}{ Sub-sample } \\
\hline & Count & Mean & $\mathrm{SD}$ & Count & Mean & SD \\
\hline Speaking proficiency & 291,135 & 0.692 & 0.462 & 229,362 & 0.700 & 0.458 \\
\hline Ethnic enclave & 290,877 & 0.123 & 0.329 & 229,104 & 0.136 & 0.342 \\
\hline Formal investment & 276,555 & 0.314 & 0.464 & 216,129 & 0.349 & 0.477 \\
\hline Informal investment & 290,853 & 0.503 & 0.500 & 229,080 & 0.554 & 0.497 \\
\hline CMA of arrival: Toronto & 291,141 & 0.484 & 0.500 & 229,368 & 0.554 & 0.497 \\
\hline CMA of arrival: Vancouver & 291,141 & 0.135 & 0.341 & 229,368 & 0.159 & 0.366 \\
\hline $\begin{array}{l}\text { Housing costs in } \mathrm{CMA} / \mathrm{CA} \text { of } \\
\text { arrival }\end{array}$ & 291,141 & 13.111 & 2.207 & 229,368 & 13.630 & 1.848 \\
\hline Ethnic share in Canada & 291,141 & 0.069 & 0.070 & 229,368 & 0.078 & 0.072 \\
\hline $\begin{array}{l}\text { Atleast a Bachelor's education } \\
\text { outside Canada }\end{array}$ & 291,141 & 0.283 & 0.451 & 229,368 & 0.307 & 0.461 \\
\hline $\begin{array}{l}\text { Formal pre-immigration } \\
\text { investment }\end{array}$ & 291,066 & 0.744 & 0.436 & 229,368 & 0.807 & 0.395 \\
\hline
\end{tabular}


MONTEIRO Cultural Assimilation: Learning and Sorting

Table 9: Summary statistics.

\begin{tabular}{|c|c|c|c|c|c|c|}
\hline \multirow[b]{2}{*}{ Variable Name } & \multicolumn{3}{|c|}{ Full sample } & \multicolumn{3}{|c|}{ Sub-sample } \\
\hline & Count & Mean & SD & Count & Mean & SD \\
\hline $\begin{array}{l}\text { Informal pre-immigration } \\
\text { investment }\end{array}$ & 291,066 & 0.262 & 0.440 & 229,368 & 0.290 & 0.454 \\
\hline Family/friend networks & 291,141 & 0.818 & 0.386 & 229,368 & 0.832 & 0.374 \\
\hline Age at immigration & 291,141 & 35.307 & 12.688 & 229,368 & 35.395 & 12.847 \\
\hline Male & 291,141 & 0.494 & 0.500 & 229,368 & 0.491 & 0.500 \\
\hline Married & 291,141 & 0.760 & 0.427 & 229,368 & 0.765 & 0.424 \\
\hline Employed & 290,294 & 0.491 & 0.500 & 228,715 & 0.497 & 0.500 \\
\hline Savings brought from outside & 275,625 & 6.311 & 4.560 & 217,260 & 6.227 & 4.617 \\
\hline Canada & & & & & & \\
\hline Family class & 291,141 & 0.328 & 0.470 & 229,368 & 0.348 & 0.476 \\
\hline Number of immigrating members & 291,141 & 2.718 & 1.541 & 229,368 & 2.782 & 1.549 \\
\hline Number of joining members & 291,141 & 0.626 & 1.222 & 229,368 & 0.671 & 1.288 \\
\hline Number of household members & 291,141 & 3.833 & 1.723 & 229,368 & 3.981 & 1.753 \\
\hline Number of children & 291,141 & 0.662 & 0.902 & 229,368 & 0.679 & 0.908 \\
\hline Months since arrival & 291,141 & 27.554 & 17.454 & 229,368 & 27.558 & 17.456 \\
\hline RGDP in place of birth & 291,141 & 0.847 & 0.899 & 229,368 & 0.882 & 0.911 \\
\hline Population in place of birth & 291,141 & 10.113 & 14.104 & 229,368 & 11.459 & 14.623 \\
\hline $\begin{array}{l}\text { Distance from capital city in } \\
\text { place of birth }\left(\times 1000^{\prime} s\right)\end{array}$ & 291,141 & 10.080 & 2.751 & 229,368 & 10.646 & 2.307 \\
\hline $\begin{array}{l}\text { Linguistic distance in place of } \\
\text { birth }\end{array}$ & 291,141 & 0.864 & 0.194 & 229,368 & 0.897 & 0.135 \\
\hline
\end{tabular}

Note 1: Sub-sample of 18-64 year old from non-English speaking households whose mother tongue is not English, and do not reside in Quebec

Note 2: All variables are weighted using the weights provided with the LSIC by Statistics Canada. 
Review of Economic Analysis 13 (2021) 115-156

Table 10: OLS estimation results of English speaking proficiency: households whose mother tongue is not English and do not reside in Quebec.

\begin{tabular}{|c|c|c|c|c|c|c|}
\hline $\begin{array}{l}\text { Dependent variable: } \\
\text { English speaking } \\
\text { proficiency }\end{array}$ & Pooled & Pooled & Pooled & $\begin{array}{c}\text { Pooled, } \\
\text { sub-sample }\end{array}$ & Pooled & Pooled IV \\
\hline RGDP per capita $(h)$ & $\begin{array}{c}0.0479 * * * \\
(0.0135)\end{array}$ & $\begin{array}{l}0.0407 * * \\
(0.0141)\end{array}$ & $\begin{array}{l}0.0399 * * \\
(0.0141)\end{array}$ & $\begin{array}{c}0.0395 \\
(0.0202)\end{array}$ & $\begin{array}{l}0.0424 * * \\
(0.0142)\end{array}$ & $\begin{array}{l}0.0678 * * \\
(0.0231)\end{array}$ \\
\hline Network $(\beta)$ & & & $\begin{array}{l}-0.0227 \\
(0.0131)\end{array}$ & $\begin{array}{l}-0.00591 \\
(0.0218)\end{array}$ & $\begin{array}{l}-0.0199 \\
(0.0130)\end{array}$ & $\begin{array}{c}0.0124 \\
(0.0236)\end{array}$ \\
\hline Ethnic enclave $(\zeta)$ & & & & & $\begin{array}{c}-0.0984 * * * \\
(0.0161)\end{array}$ & $\begin{array}{l}-1.033^{*} \\
(0.406)\end{array}$ \\
\hline Informal investment $(\iota)$ & & $\begin{array}{c}-0.0465 * * * \\
(0.0102)\end{array}$ & $\begin{array}{c}-0.0459 * * * \\
(0.0102)\end{array}$ & $\begin{array}{l}-0.0416^{*} \\
(0.0172)\end{array}$ & $\begin{array}{c}-0.0447 * * * \\
(0.0101)\end{array}$ & $\begin{array}{l}-0.0321^{*} \\
(0.0151)\end{array}$ \\
\hline Formal investment ( $\iota)$ & & $\begin{array}{c}-0.0648 * * * \\
(0.0131)\end{array}$ & $\begin{array}{c}-0.0652 * * * \\
(0.0130)\end{array}$ & $\begin{array}{c}-0.0875^{* * *} * \\
(0.0205)\end{array}$ & $\begin{array}{c}-0.0666 * * * \\
(0.0130)\end{array}$ & $\begin{array}{c}-0.0834 * * * \\
(0.0191)\end{array}$ \\
\hline $\begin{array}{l}\text { Formal pre-immigration } \\
\text { experience }(\omega)\end{array}$ & & $\begin{array}{l}0.195 * * * \\
(0.0158)\end{array}$ & $\begin{array}{l}0.195 * * * \\
(0.0158)\end{array}$ & $\begin{array}{l}0.104 * * * \\
(0.0241)\end{array}$ & $\begin{array}{l}0.194 * * * \\
(0.0158)\end{array}$ & $\begin{array}{l}0.181 * * * \\
(0.0223)\end{array}$ \\
\hline $\begin{array}{l}\text { Informal pre-immigration } \\
\text { experience }(\omega)\end{array}$ & & $\begin{array}{l}0.0881 * * * \\
(0.0116)\end{array}$ & $\begin{array}{c}0.0876^{* * * *} \\
(0.0116)\end{array}$ & $\begin{array}{c}0.0645^{* * * *} \\
(0.0188)\end{array}$ & $\begin{array}{l}0.0851 * * * \\
(0.0116)\end{array}$ & $\begin{array}{c}0.0652 * * * \\
(0.0196)\end{array}$ \\
\hline $\begin{array}{l}\text { Share of co-ethnic group in } \\
\text { Canada }(m)\end{array}$ & $\begin{array}{c}0.379 \\
(0.257)\end{array}$ & $\begin{array}{l}-0.0390 \\
(0.247)\end{array}$ & $\begin{array}{l}-0.0322 \\
(0.246)\end{array}$ & $\begin{array}{c}-2.782 * * * \\
(0.612)\end{array}$ & $\begin{array}{l}0.0901 \\
(0.246)\end{array}$ & $\begin{array}{c}1.210 \\
(0.628)\end{array}$ \\
\hline Population level $(L)$ & $\begin{array}{c}-0.00719 * * * \\
(0.00142)\end{array}$ & $\begin{array}{c}-0.00495 * * * \\
(0.00138)\end{array}$ & $\begin{array}{c}-0.00496 * * * \\
(0.00138)\end{array}$ & $\begin{array}{c}0.00620 \\
(0.00638)\end{array}$ & $\begin{array}{c}-0.00529 * * * \\
(0.00138)\end{array}$ & $\begin{array}{c}-0.00831^{* *} \\
(0.00255)\end{array}$ \\
\hline Geographic distance $(\mu)$ & $\begin{array}{l}-0.00244 \\
(0.00337)\end{array}$ & $\begin{array}{l}-0.00820 * \\
(0.00328)\end{array}$ & $\begin{array}{l}-0.00800 * \\
(0.00329)\end{array}$ & $\begin{array}{l}0.000995 \\
(0.00434)\end{array}$ & $\begin{array}{l}-0.00760 * \\
(0.00327)\end{array}$ & $\begin{array}{l}-0.00377 \\
(0.00443)\end{array}$ \\
\hline Linguistic distance $(\theta)$ & $\begin{array}{c}0.0297 \\
(0.0561)\end{array}$ & $\begin{array}{c}0.0519 \\
(0.0530)\end{array}$ & $\begin{array}{c}0.0505 \\
(0.0530)\end{array}$ & $\begin{array}{c}-0.350 * * * \\
(0.104)\end{array}$ & $\begin{array}{c}0.0515 \\
(0.0528)\end{array}$ & $\begin{array}{c}0.0624 \\
(0.0672)\end{array}$ \\
\hline Months since arrival & $\begin{array}{l}-0.0115^{*} \\
(0.00509)\end{array}$ & $\begin{array}{l}-0.0103^{*} \\
(0.00504)\end{array}$ & $\begin{array}{l}-0.0103^{*} \\
(0.00504)\end{array}$ & $\begin{array}{c}-0.0143 \\
(0.00850)\end{array}$ & $\begin{array}{l}-0.0103 * \\
(0.00503)\end{array}$ & $\begin{array}{c}-0.0121 \\
(0.00673)\end{array}$ \\
\hline $\begin{array}{l}\text { Time-varying controls } \\
\left(X_{i s t}\right)\end{array}$ & $\checkmark$ & $\checkmark$ & $\checkmark$ & $\checkmark$ & $\checkmark$ & $\checkmark$ \\
\hline $\begin{array}{l}\text { Time-invariant controls } \\
\left(X_{i s}\right)\end{array}$ & $\checkmark$ & $\checkmark$ & $\checkmark$ & $\checkmark$ & $\checkmark$ & $\checkmark$ \\
\hline Survey wave controls $(t)$ & $\checkmark$ & $\checkmark$ & $\checkmark$ & $\checkmark$ & $\checkmark$ & $\checkmark$ \\
\hline $\mathrm{N}$ & 216,672 & 204,122 & 204,122 & 78,405 & 203,858 & 203,858 \\
\hline
\end{tabular}

Note 1: All models are weighted due to Statistics Canada's RDC disclosure process.

Note 2: Cluster robust standard errors in parentheses.

Note 3: The sub-sample excludes new immigrants from source countries that scored

"medium" or "high" on the EF EPI (2017). 